\title{
Achilles and tail tendons of perlecan exon 3 null heparan sulphate deficient mice display surprising improvement in tendon tensile properties and altered collagen fibril organisation compared to C57BL/6 wild type mice
}

Cindy C Shu ${ }^{1}$, Margaret M Smith ${ }^{1}$ ， Richard C Appleyard ${ }^{2,3}$, Christopher B Little ${ }^{1,4}$, James Melrose ${ }^{\text {Corresp. }}$ 1,4,5

1 Raymond Purves Bone and Joint Laboratory, Kolling Institute of Medical Research, University of Sydney

2 Murray Maxwell Biomechanics Laboratory, Royal North Shore Hospital, University of Sydney, St. Leonards, New South Wales, Australia

${ }^{3}$ Surgical Skills Laboratory, Australian School of Advanced Medicine, Macquarie University, Sydney, New South Wales, Australia

${ }^{4}$ Sydney Medical School, Northern, University of Sydney, Sydney, Australia

5 Graduate School of Biomedical Engineering, University of New South Wales, St. Leonards, New South Wales, Australia

Corresponding Author: James Melrose

Email address: james.melrose@sydney.edu.au

The aim of this study was to determine the role of the perlecan (Hspg2) heparan sulphate (HS) side chains on cell and matrix homeostasis in tail and Achilles tendons in 3 and 12 week old Hspg2 exon 3 null HS deficient (Hspg2 $2^{\Delta 3-/ 13-}$ ) and C57 BL/6 Wild Type (WT) mice. Perlecan has important cell regulatory and matrix organizational properties through HS mediated interactions with a range of growth factors and morphogens and with structural extracellular matrix glycoproteins which define tissue function and allow the resident cells to regulate tissue homeostasis. It was expected that ablation of the HS chains on perlecan would severely disrupt normal tendon organization and functional properties and it was envisaged that this study would better define the role of HS in normal tendon function and in tendon repair processes. Tail and Achilles tendons from each genotype were biomechanically tested (ultimate tensile stress [UTS], tensile modulus [TM]) and glycosaminoglycan (GAG) and collagen (hydroxyproline) compositional analyses were undertaken. Tenocytes were isolated from tail tendons from each mouse genotype and grown in monolayer culture. These cultures were undertaken in the presence of FGF-2 to assess the cell signaling properties of each genotype. Total RNA was isolated from 3-12 week old tail and Achilles tendons and qRT-PCR was undertaken to assess the expression of the following genes Vcan, Bgn, Dcn, Lum, Hspg2, Ltbp1, Ltbp2, Eln and Fbn1. Type VI collagen and perlecan were immunolocalised in tail tendon and collagen fibrils were imaged using transmission electron microscopy (TEM). FGF-2 stimulated tenocyte monolayers displayed elevated Adamts4, Mmp2, 3, 13 mRNA levels compared to WT mice.. Non stimulated tendon Col1A1, Vcan, Bgn, Dcn, Lum, Hspg2, Ltbp1, Ltbp2, Eln and Fbn1 mRNA levels showed no major differences between the two genotypes other than a 
decline with ageing while LTBP2 expression increased. Eln expression also declined to a greater extent in the perlecan exon 3 null mice $(P<0.05)$. Type $\mathrm{VI}$ collagen and perlecan were immunolocalised in tail tendon and collagen fibrils imaged using transmission electron microscopy (TEM). This indicated a more compact form of collagen localization in the perlecan exon 3 null mice. Collagen fibrils were also smaller by TEM, which may facilitate a more condensed fibril packing accounting for the superior UTS displayed by the perlecan exon 3 null mice. The amplified catabolic phenotype of $H s p g 2^{\Delta 3-/ \Delta 3-}$ mice may account for the age-dependent decline in GAG observed in tail tendon over 3 to 12 weeks. After Achilles tenotomy Hspg $2^{\Delta-/ \Delta 3-}$ and WT mice had similar rates of recovery of UTS and TM over 12 weeks post operatively indicating that a deficiency of HS was not detrimental to tendon repair. 
Achilles and Tail Tendons of Perlecan Exon 3 Null Heparan

Sulphate Deficient Mice Display Surprising Improvement in

Tendon Tensile Properties and Altered Collagen

Fibril Organisation Compared to C57BL/6 Wild Type Mice

Cindy C Shu ${ }^{1}$, Margaret M Smith ${ }^{1}$, Richard C Appleyard ${ }^{2,3}$, Christopher B Little ${ }^{1,4} \Upsilon^{\top} J_{a m e s}$ Melrose $1,4,5$

${ }^{1}$ Raymond Purves Bone and Joint Research Laboratory, Kolling Institute of Medical Research, Northern Sydney Area Health Authority, Royal North Shore Hospital, St. Leonards, NSW 2065, Australia. ${ }^{2}$ Murray Maxwell Biomechanics Laboratory, The Institute of Bone and Joint Research, University of Sydney at the Royal North Shore Hospital, St. Leonards, NSW 2065, Australia. ${ }^{3}$ Surgical Skills Laboratory, Australian School of Advanced Medicine, Macquarie University, NSW 2109, Australia. ${ }^{4}$ Sydney Medical School, Northern, Royal North Shore Hospital, St. Leonards, NSW 2065, Australia. ${ }^{5}$ Graduate School of Biomedical Engineering, The University of New South Wales, Sydney, NSW 2052, Australia.

ๆ Corresponding author Email: james.melrose@sydney.edu.au 

ABSTRACT.

The aim of this study was to determine the role of the perlecan (Hspg2) heparan sulphate (HS) side chains on cell and matrix homeostasis in tail and Achilles tendons in 3 and 12 week old Hspg2 exon 3 null HS deficient (Hspg2 ${ }^{\Delta 3-/ \Delta 3-}$ ) and C57 BL/6 Wild Type (WT) mice. Perlecan has important cell regulatory and matrix organizational properties through HS mediated interactions with a range of growth factors and morphogens and with structural extracellular matrix glycoproteins which define tissue function and allow the resident cells to regulate tissue homeostasis. It was expected that ablation of the HS chains on perlecan would severely disrupt normal tendon organization and functional properties and it was envisaged that this study would better define the role of HS in normal tendon function and in tendon repair processes.

Tail and Achilles tendons from each genotype were biomechanically tested (ultimate tensile stress [UTS], tensile modulus [TM]) and glycosaminoglycan (GAG) and collagen (hydroxyproline) compositional analyses were undertaken. Tenocytes were isolated from tail tendons from each mouse genotype and grown in monolayer culture. These cultures were undertaken in the presence of FGF-2 to assess the cell signaling properties of each genotype. Total RNA was isolated from 3-12 week old tail and Achilles tendons and qRTPCR was undertaken to assess the expression of the following genes Vcan, Bgn, Dcn, Lum, Hspg2, Ltbp1, Ltbp2, Eln and Fbn1. Collagen fibrils were imaged using transmission electron microscopy (TEM).

FGF-2 stimulated tenocyte monolayers displayed elevated Adamts4, Mmp2, 3, $13 \mathrm{mRNA}$ levels compared to WT mice. Non stimulated tendon CollA1, Vcan, Bgn, Dcn, Lum, Hspg2, Ltbpl Eln and Fbn1 mRNA levels showed no major differences between the two genotypes other than a decline with ageing while Ltbp 2 expression increased. Eln expression also declined to a greater extent in the perlecan exon 3 null mice $(P<0.05)$. Collagen fibrils imaged using transmission electron microscopy (TEM) indicated a more compact form of collagen fibril packing in the perlecan exon 3 null mice. Collagen fibrils were smaller by TEM, which may facilitate a more condensed fibril packing accounting for the superior UTS displayed by the perlecan exon 3 null mice.

The amplified catabolic phenotype of $H s p g 2^{\Delta 3-/ \Delta 3-}$ mice may account for the age-dependent decline in GAG observed in tail tendon over 3 to 12 weeks. After Achilles tenotomy Hspg $2^{\Delta 3-/ \Delta 3-}$ and WT mice had similar rates of recovery of UTS and TM over 12 weeks post operatively indicating that a deficiency of HS was not detrimental to tendon repair. Ablation of perlecan HS moderately increased the tensile properties of tendons possibly by facilitating an increased packing density of collagen fibrils. 
65

66

67

68

69

70

71

72

73

74

75

76

77

78

79

80

81

82

83

84 85

\section{Introduction}

Heparan sulphate (HS) is an ancient glycosaminoglycan (GAG) which has evolved over hundreds of millions of years of vertebrate and invertebrate evolution (Yamada et al. 2011). HS has developed important cell regulatory and interactive properties with matrix components which stabilize the extracellular matrix (ECM) and maintain tissue homeostasis (Whitelock \& Iozzo 2005). HS is attached to a number of matrix and cell associated proteoglycans (PGs) including, perlecan, agrin, type XVIII collagen, syndecan and glypican (Gallagher 2015; Iozzo \& Schaefer 2015). Perlecan is an important matrix organizational, stabilizing and cell-signaling hub in tissues. Besides its biodiverse range of interactive ECM components perlecan-HS also binds and delivers a number of growth factors such as fibroblast growth factor (FGF)-2, 7, 9, 18; platelet derived growth factor (PDGF); Wnt (a condensation of terms describing the Winged and Int transcription factor morphogens); Sonic Hedgehog $(\mathrm{SHH})$; vascular endothelial growth factor (VEGF), and bone morphogenetic proteins (BMPs) to their cognate receptors (Whitelock et al. 2008). The aim of the present study was to ablate these HS chains by deletion of exon 3 of perlecan core protein and determine what effect this had on the homeostasis and function of tendon. A level of redundancy is normally evident in physiological systems thus we envisaged that we should also see what other molecules assisted the HS chains of perlecan in such processes which maintain tissue functionality and homeostasis. As already noted, HS also occurs on a number of proteoglycans other than perlecan however it is not known to what extent these can fill-in for a deficit in the perlecanHS chains. Our experimental design also allowed us to ascertain what accessory roles these may have in the maintenance of tissue function and homeostasis. 
Tendons are tough semi-elastic force transmitting cable-like structures between bone

90 and muscle (Benjamin et al. 2008; Bey \& Derwin 2012; Wang et al. 2012a). Type I collagen

91 is the major tendon fibrillar collagen providing it with

92 mechanical strength (Screen et al. 2015). Elastin is a minor tendon component (2-4\% w/w

93 dry weight) localized around tenocytes and between collagen fascicles. (Grant et al. 2013).

94 Elastin associated microfibrillar proteins form an integrated microstructural network along

95 with perlecan and type VI collagen (Jensen et al. 2012) forming a mechanosensory system

96 whereby tenocytes perceive and respond to perturbations in their mechanical micro-

97 environments to achieve tissue homeostasis (Pang et al. 2017). Type V and XI collagen are

98 minor internal components of I/V/XI heterofibrils (Linsenmayer et al. 1993; Nurminskaya \&

99 Birk 1998; Smith et al. 2014; Wenstrup et al. 2004; Wenstrup et al. 2011). HS binding sites

100 in type $\mathrm{V}$ and $\mathrm{XI}$ collagen have roles in tendon nucleation and lateral stabilization of

101 collagen fibrils (LeBaron et al. 1989; Ricard-Blum et al. 2006). Type VI and XI collagen are

102 associated with the cell surface of intervertebral disc cells (Hayes et al. 2016) and articular

103 chondrocytes (Carvalho et al. 2006; Horikawa et al. 2004; Wilusz et al. 2014; Zelenski et al.

104 2015a; Zelenski et al. 2015b). Localisation of type XI collagen at the cell surface is HS

105 dependant (Petit et al. 1993; Smith et al. 1989). Perlecan and elastin also colocalise at the

106 cell surface of disc cells, tenocytes and chondrocytes (Hayes et al. 2011a; Hayes et al.

107 2011b; Yu \& Urban 2010) and have roles in the assembly of elastic microfibrils at the cell

108 surface which have mechanosensory and mechanotransductive functions which direct tissue

109 homeostasis (Grant et al. 2013; Hayes et al. 2016). The small leucine repeat proteoglycan

110 (SLRP) family also interact with collagen fibres (Schonherr et al. 1995; Svensson et al.

111 2000; Viola et al. 2007). The leucine rich repeat (LRR) domains of SLRPs wrap around the 
112 collagen fibre exposing their GAG side chains which interact with the SLRP-GAG chains on

113 adjacent collagen chains providing lateral stabilization (Islam et al. 2013; Kalamajski et al.

114 2007; Svensson et al. 1995). Tenocytes express the HS-proteoglycan, perlecan (Hspg2)

115 which colocalizes with type VI collagen (Hayes et al. 2016; Wilusz et al. 2012; Wilusz et al.

116 2014; Zelenski et al. 2015b). Atomic force microscopy (AFM) studies have identified

117 biomechanical roles for these pericellular components with perlecan providing a level of 118 compliancy which may be cytoprotective (Li et al. 2015; McLeod et al. 2013; McNulty \&

119 Guilak 2015; Plodinec et al. 2010; Sanchez-Adams et al. 2013; Taffetani et al. 2015; Wang

120 et al. 2012b). Perlecan is a minor proteoglycan in normal tendon but when tendon is

121 damaged such as in a rotator cuff tendinosis model (Melrose et al. 2013) the tenocytes

122 dramatically increase their production of perlecan suggesting that it participates in tissue

123 repair. In the present study we were interested in ascertaining how ablation of the HS chains

124 in perlecan of Hspg2 exon 3 null mice affected tendon organization and functional 125 properties. We hypothesized that HS deficient tendons should be less capable of undergoing 126 effective repair when challenged by a traumatic insult (tenotomy) due to an inability of the 127 mutant perlecan from participating in HS dependent interactions with growth factors such as 128 FGF-2 to promote reparative cell proliferation and matrix synthesis as has been shown in 129 impaired vascular wound healing in Hspg2 exon 3 null mice (Zhou et al. 2004). Hspg2 exon

1303 null mice also lay down significantly lower levels of TGF- $\beta$ in tissues thus this important 131 anabolic growth factor is unavailable to participate in such tissue repair processes in this 132 genotype (Shu et al. 2016).

133 
In the present study we examined murine tail and Achilles tendon from C57BL/6 and

135 Hspg2 exon 3 null mice employing biomechanical, biochemical and molecular methods and

136 imaged tendons by immunolocalising type VI collagen and perlecan and collagen fibril

137 organization by transmission electron microscopy (TEM).

\section{Materials and Methods}

139 Ethics approval for this study was obtained from The Animal Care and Ethics Review Board of

140 The Royal North Shore Hospital, St. Leonards, Sydney, Australia. (RNS/UTS 0709-035A J

141 Melrose, C Little, R Appleyard. Evaluation of $\triangle 3-/ \triangle 3-H S P G 2$ HS deficient mice).

142 Tissues

143 Hspg2 $2^{\Delta 3-/ \Delta 3-}$ homozygous mouse breeding pairs backcrossed into a C57BL/6 background for

14412 generations were kindly supplied by Dr R Soinninen, University of Oulu BioCentre, (Oulu,

145 Finland). WT C57BL/6 mice were obtained from Jackson Laboratories (Bar Harbor, Maine,

146 USA). All mice were caged in groups ( $\mathrm{n}=2-5$ mice per $500 \mathrm{~cm}^{2}$ cage floor space) and received

147 acidified water and complete pelleted food ad libitum. All cages were individually ventilated

148 with filter lids, sterilised Aspen chip bedding, environmental enrichment (tissues, house) and 149 maintained at $20-22^{\circ} \mathrm{C}, 50-60 \%$ humidity, with a 12 hour light-dark cycle regimen. Only male 150 mice were used for these studies.

151

152 Overview of the analyses undertaken in this study.

153 1. Genotyping of WT and Perlecan exon 3 null mice.

154 2. Weight of WT and Perlecan exon 3 null mice up to 20 weeks of age.

155 3. HS contents of purified perlecan samples measured by ELISA.

156 4. Material properties (UTS/TM) of tail and Achilles tendons over 3-12 weeks of age. 
157 5. Compositional analyses of the GAG and hydroxyproline contents of tail tendons in WT and 158 Perlecan exon 3 null mice up to 12 weeks of age.

159 6. Material properties of control non operated Achilles tendons over 2-8 weeks of age and the 160 recovery of material properties (UTS/TM) of tenotomised Achilles tendons in WT and 161 Perlecan exon 3 null mice over 2-8 weeks post operatively.

162 7. Collagen I, versican, biglycan, lumican, decorin, perlecan, LTBP-1 and 2, fibrillin-1, elastin 163 gene expression by 3-12 week old WT and Perlecan exon 3 null tail tenocytes .

164 8. Response of WT and Perlecan exon 3 null mouse tail tenocyte monolayer cultures to FGF-2 165 (1-100 ng/ml): Mmp2, Mmp3, Mmp13, Adamts4, Timp2, Timp3 gene expression.

166 9. Transmission electron microscopy of collagen fibril cross-sectional areas in tail and 167 Achilles tendon in WT and Perlecan exon 3 null mice at 3 and 12 weeks of age.

168 Genotyping of Hspg2 $2^{\Delta 3-/ \Delta 3-}$ mice

169 Genomic DNA was extracted from WT and Hspg $2^{\Delta 3-/ \Delta 3-}$ mouse tail tips using commercial kits

170 (Qiagen). Specific regions of the mouse perlecan gene were amplified by PCR using 171 genotyping primers recognising intron 2 of mouse Hspg2 (GTA GGG ACA CTT GTC ATC $172 \mathrm{CT}$ ), exon 3 (CTG CCA AGG CCA TCT GCA AG) and Hspg2 ${ }^{43-/ \Delta 3-}$ (AGG AGT AGA AGG 173 TGG CGC GAA GG). The PCR products were identified by electrophoretic separation on $2 \%$ 174 w/v agarose gels (Supplement Figure 1A). 175

176 Weights of WT and Perlecan exon 3 null mice.

177 Mouse weights were measured in each genotype over 10-20 weeks of age (Fig 1B). 178

179 Isolation and identification of perlecan from skeletal muscle. 
180 Muscle from the hind limbs of two WT and two Hspg2 exon 3 null mice were finely minced

181 and extracted with $6 \mathrm{M}$ urea $50 \mathrm{mM}$ Tris- $\mathrm{HCl} \mathrm{pH} 7.2(15 \mathrm{ml} / \mathrm{g}$ tissue $)$ for $48 \mathrm{~h}$ at $4^{\circ} \mathrm{C}$. Perlecan

182 was isolated using Resource Q anion exchange FPLC and electrophoresed on pre-poured 3-8\%

183 PAG Tris-acetate gradient gels, blotted to nitrocellulose and perlecan identified using MAb

184 H300 (Santa-Cruz) (Supplement Fig1B). The GAG side chains of these samples were

185 analysed by ELISA using MAb 10-E-4 and 3-G-10. Selected samples were pre-digested with

186 Heparitinase III to generate $\Delta$-HS stub epitopes reactive with MAb 3-G-10.

187

188

Biomechanical assessment of murine tail and Achilles tendons

189 Tail tendons from 3, 6 and 12 week old mice, $(n=6-8$ at each age point), were dissected from

190 underlying bone and connective tissue within $1 \mathrm{~h}$ of death. Mouse tails contain two dorsal and 2

191 ventral tendons. Ventral tendons were used exclusively in this study. These were dissected out

192 approximately $5 \mathrm{~mm}$ from the base of the tail. Segments of 20-30 mm were used for

193 biomechanical testing. A single ventral tendon was used for biomechanical testing per animal.

194 The cross-sectional area of the tendon was measured using a custom micrometer device (Choi et 195 al. 2016). Achilles tendons attached to the calcaneous and gastrocnemius muscles proximally

196 were also collected. Biomechanical testing was undertaken in an Instron 8874 servo-hydraulic

197 material testing apparatus. Tail tendons were marked with two reference points using Alcian blue

198 and anchored in custom-built brass clamps. The distance between the clamps was 10mm. With

199 Achilles tendons the calcaneous was anchored in the lower clamp, and gastrocnemius muscle

200 attached to the upper clamp. Tendons were loaded to failure at a rate of $1 \mathrm{~mm} / \mathrm{second}(10 \%$

201 strain), real-time videos of each test were recorded at 100 frames per second using a high-speed

202 camera mounted perpendicular to the tendon (Marlin F/145B camera, Allied Vision Technology, 
203 Massachusetts, USA). The videos of tendon deformation were analysed using LabView software

204 (National Instruments, Austin, Texas, USA) and the data normalised to tendon cross-sectional

205 area to calculate stress. Elastic modulus was calculated from the gradient of the linear region on

206 the stress-strain curve.

207

208 Tendon compositional analyses

209 Finely-diced tail tendons were papain digested and sulfated GAG determined using 1,9-

210 dimethylmethylene blue, bovine tracheal CS was used as standard (Farndale et al. 1986).

211 Aliquots of the tissues were also hydrolysed in $6 \mathrm{M} \mathrm{HCl}$ for hydroxyproline determinations by

212 the dimethylaminobenzaldehyde procedure (Stegemann \& Stalder 1967).

213

214 Achilles tendon tenotomy

215 Under general anaesthesia (2\% isofluorane) Achilles tendons of 12 week old mice from each

216 genotype were sharply transected mid-way between the calcaneal attachment and muscle

217 leaving the plantaris tendon intact. The skin incision was closed with a subcutaneous Vicryl

218 8/0 suture and sealed with cyanoacrylate tissue glue. Mice were returned to their pre-operative

219 social groups post-tenotemy. Groups of mice ( $\mathrm{n}=10$ for each time point) were sacrificed at 2, 4

220 or 8 weeks post operatively (PO) and Achilles material properties measured.

221

222 Gene expression in tail tendons

223 Mouse tail tendons from 3, 6, and 12 week old WT and $H \operatorname{spg} 2^{\Delta 3-/ \Delta 3-}$ mice were pooled ( $\mathrm{n}=6$ at

224 each age point) to provide $\sim 50 \mathrm{mg}$ wet weight of tissue for each RNA isolation. Tendons were

225 snap frozen in liquid nitrogen, freeze-shattered using a Mikro dismembranator (B. Braun 
226 Biotech International, Melsungen, Germany) and total RNA extracted using Trizol (Invitrogen,

227 Mulgrave, VIC, Australia), purified using Qiagen RNeasy columns (Qiagen, Chadstone

228 Centre, VIC, Australia) and quantified by NanoDrop (ThermoFisher Scientific, Scoresby, VIC,

229 Australia). RNA (1 $1 \mu \mathrm{g})$ from each sample was reverse transcribed using Omniscript Reverse

230 Transcription Kit (Qiagen) with random pentadecamers (50ng/ml, Sigma-Genosys, Castle Hill,

231 NSW, Australia) and RNase inhibitor (10U per reaction, Bioline, Sydney, NSW, Australia).

232 The cDNA was subjected to qRT-PCR in a Rotorgene 6000 (Qiagen) using Immomix

233 (Bioline, Sydney, NSW, Australia), SYBR Green I (Cambrex Bioscience, Rockland, ME,

234 USA), and $0.3 \mu \mathrm{M}$ validated murine-specific primers. Relative copy numbers for genes of

235 interest were determined using a standard curve generated from pooled cDNA normalised to

236 Gapdh. PCR primer specificity was confirmed by sequencing (SUPAMAC, Sydney

237 University). Genes, primers, and annealing temperatures are listed in Table 1.

238

239 Tenocyte monolayer cultures stimulated with FGF-2.

240 Three week old tail tendons from six mice of each genotype were macerated and cultured in

$2412 \mathrm{ml} \mathrm{DMEM} / 10 \% \mathrm{FBS} / 2 \mathrm{mM}$ L-glutamine under an atmosphere of $5 \% \mathrm{CO}_{2}$, with media

242 changes every 3-4 days. After 2 weeks the tissue was removed and the attached cells detached

243 with trypsin-EDTA and sub-cultured in fresh media. Passage 3 cells were cryopreserved $\left(10^{7}\right.$

244 cells $/ \mathrm{ml}, 0.5 \mathrm{ml}$ aliquots) in 10\% v/v DMSO, 20\% v/v FBS in DMEM. Tenocytes were re-

245 seeded in 6-well plates at $2 \times 10^{5}$ cells per well for $48 \mathrm{~h}$. The cultures were washed three times

246 in serum-free DMEM, and incubated in DMEM/1\% v/v FBS containing $0,1,10$ or $100 \mathrm{ng} / \mathrm{ml}$

247 FGF-2 (PeproTech Inc, Rocky Hill, NJ, USA) for 24 h. Total RNA was extracted using 
248 Trizol, $1 \mu \mathrm{g}$ RNA was reverse-transcribed then qRT-PCR undertaken for $M m p 2, M m p 3$,

249 Mmp13, Timp1, Timp3 and Adamts4.

250

251 Transmission electron microscopy of tail and Achilles tendon

252 Three tendons from 3- and 12-week old WT and Hspg2 $2^{\Delta 3-/ \Delta 3-}$ mice were washed in $0.1 \mathrm{M}$ 253 sodium cacodylate buffer containing $3 \mathrm{mM} \mathrm{CaCl}_{2}, 100 \mathrm{mM}$ sucrose $\mathrm{pH} 7.4$ and fixed in $2.5 \%$ $254 \mathrm{v} / \mathrm{v}$ glutaraldehyde $/ 0.5 \% \mathrm{v} / \mathrm{v}$ paraformaldehyde for $30 \mathrm{~min}$ at room temperature, $4^{\circ} \mathrm{C}$ for $24 \mathrm{~h}$, 255 followed by storage in $70 \% \mathrm{v} / \mathrm{v}$ ethanol. The fixed tissues were trimmed and post-fixed in $2 \%$ $256 \mathrm{w} / \mathrm{v} \mathrm{OsO}_{4}$ in $0.1 \mathrm{M}$ cacodylate buffer for $1-2 \mathrm{~h}$ at $4{ }^{\circ} \mathrm{C}$ followed by dehydration in serial graded 257 ethanol washes $(25 \%, 50 \%, 75 \%, 95 \%, 100 \%, 100 \%$, all v/v). The tissues were infiltrated with 258 Spurrs resin/ethanol (1:1) overnight then with two overnight infiltrations of $100 \%$ resin and 259 polymerised at $60^{\circ} \mathrm{C}$ for $48 \mathrm{~h}$. Ultra-thin transverse tissue sections $(70 \mathrm{~nm})$ were cut using an 260 Ultracut $\mathrm{T}$ microtome and transferred to copper grids (200 mesh). The specimens were 261 stained/contrasted for $10 \mathrm{~min}$ with $2 \% \mathrm{w} / \mathrm{v}$ uranyl acetate and Reynold's lead citrate (1.33g 262 lead nitrate, $1.76 \mathrm{~g}$ sodium citrate dihydrate, $5 \mathrm{ml} 1 \mathrm{M} \mathrm{NaOH}$, in $50 \mathrm{ml} \mathrm{H}_{2} \mathrm{O}$ final total volume). 263 The specimens were examined in a JEOL1400 transmission electron microscope at $120 \mathrm{kV}$ at $26425,000 \times$ magnification. The images were analysed using ImageJ (public domain Java-based 265 image processing software developed by NIH) to determine fibril diameters. Three separate 266 regions of each specimen were photographed and at least 400 fibrils were measured in each 267 image. When the fibril had a non-circular configuration the diameter across the minimum axis 268 was measured. The frequency distribution of the collagen fibril diameters was calculated as a 269 percentage of the total fibril numbers measured. 


\section{Statistical analyses}

272 Groups of paired parametric data from mechanical measurements, hydroxyproline and sGAG

273 compositional data and fibril diameters measured by TEM were compared by unpaired

274 Students-t test for differences between age and genotypes. Non-Gaussian data from qRT-PCR

275 analyses were analysed using Kruskal-Wallis then Mann-Whitney U ranked tests. The alpha

276 level was set at 0.05 .

277 


\section{$279 \quad$ Results}

$280 \quad$ Genotyping of mouse strains

281 Figure 1A depicts the replacement of perlecan exon-3 with a pGK-neo cassette in the $282 H s p g 2^{\Delta 3-/ \Delta 3-}$ mice. Hspg $2^{\Delta 3-/ \Delta 3-}$ mice were fertile and litters were of expected size. They 283 had no gross abnormalities or difference in appearance compared to WT mice at birth. By 2843 weeks of age, the previously reported micropthalia in $H s p g 2^{\Delta 3-/ \Delta 3-}$ animals was evident. 285 Age-matched Hspg2 $2^{\Delta 3-/ \Delta 3-}$ mouse body weights were less than corresponding WT mice 10 286 to 18 weeks of age (Fig 1B). Although smaller, Hspg2 ${ }^{\Delta 3-/ \Delta 3-}$ mice had similar skeletal 287 proportions to WT mice and no apparent musculoskeletal abnormalities. Mutant mice were 288 more docile when handled but no other behavioural abnormalities were noted other than 289 small leaky eyes.

290

291 Perlecan isolated from C57BL/6 Wild Type and Hspg2 exon 3 null mice.

292 Perlecan isolated from skeletal muscle demonstrated a core protein of $\sim 420 \mathrm{kDa}$ for 293 WT perlecan while mutant perlecan had a molecular weight of $\sim 300-390 \mathrm{kDa}$ (Supplement 294 Figure 1B). ELISA analysis demonstrated that perlecan from C57BL/6 Wild Type mice 295 contained HS chains while Hspg2 exon 3 null perlecan did not (Fig 1C).

\section{Tendon material properties and biochemical composition}

Ultimate tensile stress (UTS) (Fig 2A, C) and tensile modulus (TM) (Fig 2B, D) measurements of tail (Fig 2A, B) and Achilles (Fig 2C, D) 3-12 week old tendons 
301

302

303

304

305

306

307

308

309

310

311

313

314

315

316

317

318

320

321

322

323

values for tail and Achilles tendons increased with maturation (Fig 2A-D). 6-12 week old tail tendons from the perlecan exon 3 null mice displayed moderately greater UTS and TM values compared to WT tail tendons $(\mathrm{P}<0.05)$ (Fig 2A, B). Tail tendon sGAG levels underwent a maturation-dependent decline in $H_{s p g} 2^{\Delta 3-/ \Delta 3-}$ mice (Fig 2E, F), with 50\% reduction in GAG content at 12 weeks compared to 3 weeks $(3>6>12$ week), and lower GAG content in Hspg2 $2^{\Delta 3-/ \Delta 3-}$ mice whereas hydroxyproline contents did not change significantly with age or genotype.

\section{Achilles tendon tenotomy}

With tissue maturation the non-operated contralateral perlecan exon 3 null Achilles tendons displayed moderately higher UTS and TM values by 20 weeks of age compared to the WT tendons $(\mathrm{P}<0.05)$. Following tenotomy, WT and perlecan exon 3 null Achilles tendons partially recovered their UTS and TM material properties over an 8 week recovery period reaching approximately $50 \%$ of the UTS/TM values of the non-operated contralateral tendons at 20 weeks of age (Fig 3A, C). There was a moderately increased trend in the rate of recovery of UTS/TM in the WT mice compared to the perlecan exon 3 null mice following tenotomy. The UTS and TM values for the WT tendons however were more variable than the perlecan exon 3 null tendons. Statistics on the mean data at each time point for each genotype indicated this trend was only moderately elevated in the WT Achilles tendons with a $\mathrm{P}<0.05$ (Fig 3B, D).

Tail tendon gene expression

qRT-PCR of selected ECM genes in mouse tail tendons, (Collal, Vcn, Bgn, Dcn), demonstrated a maturation-dependent decline in gene expression over 3 to 12 weeks in 
324 both genotypes (Fig 4). Relative gene expression levels for perlecan core protein (Hspg2)

325 and the elastin micro-fibril associated proteins Ltbpl, Fbnl, and Eln were significantly

326 lower in Hspg $2^{\Delta 3-/ \Delta 3-}$ mice compared to age-matched WT mice. Ltbp2 gene expression

327 displayed an increase with ageing in both genotypes.

328

329

Tendon outgrowth cell responses to FGF-2

Mmp2, Mmp3, Mmp13 and Adamts4 expression were significantly higher in basal $H \operatorname{spg} 2^{\Delta 3-/ \Delta 3-}$ mice compared to WT tenocyte cultures (0ng/ml FGF-2). Mmp2 expression in $H_{s p g} 2^{\Delta 3-/ \Delta 3-}$ cultures remained significantly higher than WT at all doses of FGF-2 examined. Mmp3 and Mmp13 expression increased dose-dependently in both genotypes, and this response was greater in Hspg2 $2^{\Delta 3-/ \Delta 3-}$ tenocytes at high doses of FGF-2 (Mmp3 33-

33536 fold versus 50 fold and Mmp13 134-192 fold versus 226-248 fold at 10 and 100ng/ml in

336 WT and Hspg2 $2^{\Delta 3-/ \Delta 3}$ ) (Fig 5). Adamts4 gene expression was significantly decreased by increasing concentrations of FGF-2 treatment in Hspg2 $2^{\Delta 3-/ \Delta 3-}$ tenocyte cultures but still 338 remained significantly greater than WT cultures at all doses. FGF-2 up-regulated Timp1 339 gene expression, less so in $H s p g 2^{\Delta 3-/ \Delta 3-}$ compared to WT cultures, favoring a pro-catabolic 340 phenotype in the mutant mice. Expression of Timp3, the naturally occurring inhibitor of

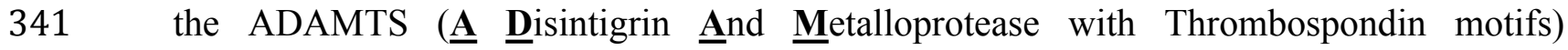
342 enzymes, while equivalent in basal culture, was decreased to a greater extent by FGF-2 in $343 \quad H s p g 2^{\Delta 3-/ \Delta 3-}$ tenocyte cultures at the highest dose. electron microscopy 
Figure 6 shows the changes in collagen fibril diameter of tail and Achilles tendon

348 measured from TEM images of 3 and 12 week old mouse tendons. In 3 week old mice

349 there were small differences between genotypes in mean fibril diameter $( \pm$ SD) in tail $($ WT

$350=202 \pm 60 \mathrm{~nm}, H \operatorname{spg} 2^{\Delta 3-/ \Delta 3-}=193 \pm 46 \mathrm{~nm}$; not significant) and Achilles $(\mathrm{WT}=160 \pm$

$\left.35144 \mathrm{~nm}, H_{s p g} 2^{\Delta 3-/ \Delta 3-}=139 \pm 37 \mathrm{~nm} ; P<0.001\right)$ tendons (Fig 6A, B). This was reflected in

352 minor differences in frequency distributions of collagen fibril diameters in the two

353 genotypes in these immature mice (Fig 6C, D). An increase in average collagen fibril

354 diameter was evident from 3 to 12 week old in the WT tail $(202 \pm 60 \mathrm{~nm}$ to $243 \pm 81 \mathrm{~nm} ; P$

$355<0.0001)$ and Achilles $(160 \pm 44 \mathrm{~nm}$ to $210 \pm 63 \mathrm{~nm} ; P<0.001)$ tendons, accompanied by

356 an increase in collagen fibril diameter distribution in both tendons (Fig 6C, D. Mean

357 Achilles collagen fibril diameter also increased from 3-12 week old in $H s p g 2^{\Delta 3-/ \Delta 3-}$ mice

$358(139 \pm 37 \mathrm{~nm}$ to $150 \pm 34 \mathrm{~nm} \mathrm{p}<0.001)$ to a lesser extent than in WT mice $(8$ versus $31 \%)$,

359 and significantly decreased in Hspg2 $2^{\Delta 3-/ \Delta 3-}$ tail tendons $(193 \pm 46 \mathrm{~nm}$ to $84 \pm 28 \mathrm{~nm} ; P<$

360 0.001). Thus differences between genotypes in mean collagen fibril diameter and

361 distribution in tail and Achilles tendons were more marked by 12 weeks of age.

362

363

364 


\section{Discussion}

366

This study uncovered some surprising findings regarding the potential role of perlecan

HS chains in the functional properties of tail and Achilles tendon. We hypothesised that ablation

368

of the HS chains of perlecan should have a significant effect on ECM organization and would also be reflected in tendon material properties. The HS chains of perlecan also interact with growth factors and present these to their cognate receptors to modulate cell signaling, proliferation, differentiation and matrix synthesis (Whitelock et al. 2008). Thus we expected that ablation of perlecan HS chains would also disrupt normal tissue homeostasis. None of these possibilities actually happened. Perlecan was selected as a target proteoglycan to investigate in this study since it is a major, ubiquitous, HS-proteoglycan with established functional attributes in connective tissues (Whitelock \& Melrose 2011). Other extracellular HS substituted proteoglycans such as agrin and type XVIII collagen and the cell associated glypican and syndecan families have also been identified (Iozzo 1998; Iozzo \& Schaefer 2015). Type XVIII collagen and the glypican family of proteoglycans have not been identified in the main substance of tendon. Agrin is an extracellular HS-proteoglycan of the muscle-tendon neuromuscular junction (NMJ) and is also found in articular cartilage (Finni 2006). FGF-2-agrin interactions modulate neurite outgrowth in the NMJ and stabilize axonal development (Cotman et al. 1999; Kim et al. 2003). Syndecan was shown to be produced by tenocytes in a proteomic study (Sato et al. 2016; Sato 2011; Smith et al. 2012), by fibroblasts (Sawaguchi et al. 2006) and chondrocytes (Melrose et al. 2012) and participates in FGF-2 mediated cell signalling (Salmivirta \& Jalkanen 1995). Members of the syndecan family and agrin are therefore candidate HS-proteoglycans which could potentially act in a "fill-in" capacity in HS deficient perlecan exon 3 null tenocytes. NG2/CSPG4 is another transmembrane proteoglycan produced by cells of a chondrogenic 
388 background such as tenocytes. CSPG4 binds FGF2 resulting in cell proliferation (Cattaruzza et

389 al. 2013; Takeuchi et al. 2016) it also acts as a type VI collagen receptor and binds to perlecan

390 (Tang et al. 2018). Type VI collagen/CSPG4 regulate tenocyte behavior, play important roles in

391 the assembly of tendon matrix, and have crucial roles in tendon repair processes (Sardone et al.

392 2016). CSPG4 is therefore another candidate proteoglycan worthy of consideration in the FGF-2

393 mediated responses we observed in the present study. However, it is not known to what extent

394 tenocyte CSPG4 undergoes cell signalling in response to FGF-2. Upon FGF binding to

395 microvascular cells, the cytoplasmic domain of CSPG4 is phosphorylated, but there is no

396 evidence that this event elicits signal transduction in a similar way to how this occurs in

397 perlecan-HS-FGF-FGFR mediated interactions (Sardone et al. 2016). It therefore remains to be

398 established to what extent CSPG participates in tenocyte responses in the present study. Wnt-

399 signalling is a highly conserved and tightly controlled cascade of signal transduction pathways,

400 essential for articular cartilage development and joint homeostasis (Chun et al. 2008). Syndecan-

$4014(\mathrm{Sdc} 4)$ is the most abundant syndecan family member in articular cartilage (Echtermeyer et al.

402 2009). Sdc4 and agrin facilitate Wnt-signalling in articular chondrocytes, but have opposing

403 actions: Sdc4 promotes Wnt signaling, reduces the expression of Acan and Col2Al and inhibits

404 GAG production (van den Berg 2011), agrin inhibits Wnt signalling and promotes

405 chondrogenesis (Eldridge et al. 2016; Hausser et al. 2007). Syndecan-4 controls the activation

406 of ADAMTS-4 and 5 either by direct interaction with these proteases or indirectly by regulation

407 of MAPK dependent synthesis of MMP-3 (Echtermeyer et al. 2009). The initiating role of

408 ADAMTS-5 in proteoglycan loss in OA appears dependent on interaction with the

409 transmembrane proteoglycan Sdc-4. Sdc-4 deficient mice are less prone to cartilage changes

410 induced by experimental OA and display reduced ADAMTS-5 activity (van den Berg 2011). 
411 Sdc4 also regulates collagen fibril architecture, condensation and orientation, proteoglycan

412 production and remodelling. Tenocytes and fibroblasts both participate in $W n t$ signaling and

413 express Wnt3a, $\beta$-catenin, Lrp5 (Low-density lipoprotein receptor-related protein 5) and

414 Tcfl(hepatocyte nuclear factor-1 alpha) (Liu et al. 2014; Liu et al. 1995; Liu et al. 2010).

415 The Wnt signalling pathway plays a vital role in pathological calcification in animal models of

416 tendinopathy and in pathological human tendon (Liu et al. 2013). Wnt3a increases ALP activity,

417 calcium nodule formation and expression of osteogenic markers in tendon derived progenitor

418 stem cells. In equine tendon, Wnt/ $\beta$-catenin signalling promotes the differentiation of bone

419 marrow stromal Mesenchymal stem cells (MSCs) into tenomodulin expressing tendon cells

420 (Miyabara et al. 2014). Wnt/ $\beta$-catenin signalling in 6 week old rat Achilles tendon however

421 suppressed expression of the tenogenic genes Scleraxis $(S c x)$, Mohawk homeobox $(M k x)$,

422 and Tenomodulin (Tnmd) suggesting that Wnt/ $\beta$-catenin signalling repressed tenogenic gene

423 expression in rats (Kishimoto et al. 2017). These two studies illustrate the need for a better

424 understanding of the regulatory mechanisms which control tendon development and remodelling

425 and the extracellular factors which control tenocyte gene expression. The observations of the

426 present study which showed that ablation of perlecan HS chains resulted in a more compactly

427 organized tendon with increased tensile properties represent a further aspect of the functional

428 organization of tendon tissue and tenocyte regulation.

429

430

The reduction in size of collagen fibrils we observed in the Hspg2 exon 3 null mouse tail

431 tendons was also an unexpected finding of the present study. In other knock-out studies where

432 proteins which regulate tendon collagen fibrillogenesis are deleted, the collagen fibrils generally

433 display an increased size (Ameye et al. 2002; Chen et al. 2003; Danielson et al. 1997; Ezura et al. 
434 2000; Jepsen et al. 2002). Collagen VI knockout mice are an exception and small collagen fibrils

435 similar in size to those observed in the present study are also observed (Alexopoulos et al. 2009).

436 Another aspect of the hypothesis we tested in this study was whether a deficiency of perlecan HS

437 chains resulted in an impaired ability of the tendons to undergo intrinsic repair processes due to

438 the lack of a contribution from HS dependent growth factors. Skin wounds display incomplete

439 vascular repair in Hspg2 exon 3 null mice (Zhou et al. 2004). Tenotimised Achilles tendons

440 from the Hspg2 perlecan exon 3 null and WT mice also displayed a similar mean recovery rate in

441 UTS and TM compared to non-operated contralateral Achilles tendons. This response was more

442 variable in the WT mice and the trend in the recovery of material properties in the Achilles

443 tendons was slightly slower in the Hspg2 exon 3 null mice compared to WT mice but this was

444 not statistically significant. Achilles tendons in both of the mouse genotypes had recovered

445 around $50 \%$ of their UTS and TM by 12 weeks post tenotomy. Thus HS is not a strict

446 requirement for tendon repair and in this respect repair processes in tendon differ from those

447 operative in the repair of skin wounds in perlecan exon 3 null mice.

448

449 Shoulder instability is a clinical problem occurring due to tendon laxity, recurrent

450 subluxations or in severe cases complete shoulder dislocation (Walz et al. 2015). This is a 451 painful condition, which increases in severity if left untreated. Treatment options include 452 surgical intervention, physiotherapy with specific strengthening exercises for muscle groups to 453 better hold the shoulder in proper position, cortisone injections and anti-inflammatory 454 medications (Bigliani et al. 1996; Moon et al. 2011). The findings of the present study indicate 455 that digestion of the HS component of shoulder tendons with heparanase may improve their 
456 tensile properties making them more supportive in cases of shoulder laxity. Future research

457 should aim to explore this possibility further.

458

459 Conclusions

460 Ablation of perlecan HS chains was not detrimental to tendon function or intrinsic repair and 461 moderately improved their tensile properties. The reduced size of collagen fibrils, and a more 462 condensed fibril packing density in HS deficient perlecan exon 3 null mice and reduced 463 expression of elastin undoubtably contributed to the observed change in tendon material 464 properties. Perlecan exon 3 null tenocytes displayed a catabolic phenotype with FGF-2. This 465 may explain why an age dependent decline in sGAG content was observed in HS-deficient 466 tendons. Agrin and syndecan-4 in tendon are putative "fill-in" proteoglycans which may have 467 acted in place of HS free perlecan. Syndecan-4 already has established roles in the regulation of 468 cartilage degradation through activation of ADAMTS-5 in OA.

469

\section{Acknowledgements}

471 Dr Joanna Peterson developed the tendon materials testing procedures used in this study as part 472 of her PhD studies at The Murray-Maxwell Biomechanics Laboratory within the Institute of 473 Bone and Joint Research of The Kolling Institute of Medical Research. Ms Susan Smith 474 expertly conducted the tendon immunolocalisations reported in this study.

475

476

477

478

479

480

481

482 
483

484

485

486

487

488

489

490

491

492

493

494

495

496

497 Legends to Figures

498 Figure 1. Genomic organisation of exons 2-5 of the WT and Hspg2 $2^{\Delta 3-/ \Delta 3-}$ mice (A). Body 499 weights of male WT ( $\square-\square)$ and $H \operatorname{spg} 2^{\Delta 3-/ \Delta 3-}$ mice ( $\left.\square-\square\right)$ from 10-20 weeks of age (B). ELISA 500 analysis of perlecan GAG side chains using MAb 10E4 to native HS and MAb $3 \mathrm{G} 10$ to the

$501 \Delta \square$ HS stub epitope generated by heparitinase III pre-digestion demonstrating an absence of HS 502 in the mutant perlecan. (C)

503 Figure 2. Tendon material properties: ultimate tensile stress $(A, C)$ and tensile modulus $(B, D)$.

504 GAG (E) and Hydroxyproline (F) content of 3 to 12 week-old WT and Hspg2 $2^{\Delta 3-/ \Delta 3-}$ tail tendons.

505 Box plots show mean (line in box) and data range (box 25-75\%, whiskers maximum-minimum).

506 White bars: WT; Gray bars: Hspg2 ${ }^{\Delta 3-/ \Delta 3-}$. Bar graph shows mean \pm standard error of mean.

507 Brackets $-P<0.05$ between samples, $* P<0.05$ between genotypes. $\mathrm{n}=6-8$ for each sample.

508 Figure 3. Achilles tendon material properties at 2, 4 and 8 weeks after surgical tenotemy: 509 normal contralateral (A, C) and surgical tenotomy (B, D), equivalent to 14, 16 and 20 weeks of 
510 age. White bars: WT; Gray bars: $H s p g 2^{\Delta 3-/ \Delta 3-}$. Box plot show median (line in box), inter-quartile

511 range (box) and data range (whiskers, maximum - minimum). Bracket $-P<0.05$ between

512 samples. $* P<0.05$ between genotypes. $\# P<0.05$ to contralateral. $\mathrm{n}=6-8$.

513 Figure 4. Comparative gene expression of selected extracellular matrix genes and elastin-

514 associated protein genes in mouse ex vivo tail tendons at 3, 6 and 12 weeks old. * $P<0.05$

515 between genotype. Data were normalised to Gapdh expression. White bars: WT; Gray bars:

$516 H_{s p g} 2^{\Delta 3-/ \Delta 3-}$. Box plot shows mean (line in box), interquartile range (box) and data range

517 (whiskers, maximum - minimum). $\mathrm{n}=6$ per sample.

518 Figure 5. Gene expression in 3 week-old mouse tail tendon outgrowth tenocytes cultured with

519 FGF-2 $(0,1,10$ or $100 \mathrm{ng} / \mathrm{ml})$. Data expressed as a fold change relative to the expression of the

520 WT control (0 ng/ml FGF-2). White bars: WT; Gray bars: Hspg2 $2^{\Delta 3-/ \Delta 3-}$. Box plot shows mean

521 (line in box), interquartile range (box) and maximum - minimum (whiskers). $\mathrm{n}=6$ for each

522 sample. \# $P<0.05$ compared to untreated control; * $P<0.05$ between genotypes at that

523 concentration of FGF-2. Note Mmp13 Y-axis differs from all other genes with range from 0.1-

$52410000 x$ instead of $0.1-100 x$.

525 Figure 6. Measurement of the collagen fibril diameters in cross-section of 3 and 12 week-old

526 mouse tail and Achilles tendons from transmission electron microscopy (TEM) images. A total

527 of 400 fibrils from each image were measured in each case. Representative TEM images (A, B)

528 are shown with respective mean \pm standard deviations. Fibril diameter data were categorised

529 into defined nanometer ranges ( $\mathrm{x}$ axis) giving the overall size distribution $(\mathrm{C}, \mathrm{D})$. The

530 frequencies were expressed as a percentage of the total fibril numbers counted (y axis). Three

531 tendon samples from three animals per age/genotype were measured. Bar graph shows mean \pm

532 standard deviation. White bars: WT; Gray bars: Hspg2 $2^{\Delta 3-/ \Delta 3-}$. 
533

PeerJ reviewing PDF | (2018:02:25075:2:1:NEW 2 Jun 2018) 


\section{BIBLIOGRAPHY}

535

536

537

538

539

540

541

542

543

544

545

546

547

548

549

550

551

552

553

554

555

556

557

558

559

560

561

562

563

564

565

566

567

568

569

570

571

572

573

574

575

576

577

578

579
Alexopoulos LG, Youn I, Bonaldo P, and Guilak F. 2009. Developmental and osteoarthritic changes in Col6a1-knockout mice: biomechanics of type VI collagen in the cartilage pericellular matrix. Arthritis Rheum 60:771-779. 10.1002/art.24293 [doi]

Ameye L, Aria D, Jepsen K, Oldberg A, Xu T, and Young MF. 2002. Abnormal collagen fibrils in tendons of biglycan/fibromodulin-deficient mice lead to gait impairment, ectopic ossification, and osteoarthritis. FASEB J 16:673-680. 10.1096/fj.01-0848com [doi]16/7/673 [pii]

Benjamin M, Kaiser E, and Milz S. 2008. Structure-function relationships in tendons: a review. $J$ Anat 212:211-228. 10.1111/j.1469-7580.2008.00864.x

Bey MJ, and Derwin KA. 2012. Measurement of in vivo tendon function. J Shoulder Elbow Surg 21:149-157. 10.1016/j.jse.2011.10.023

Bigliani LU, Kelkar R, Flatow EL, Pollock RG, and Mow VC. 1996. Glenohumeral stability. Biomechanical properties of passive and active stabilizers. Clin Orthop Relat Res:13-30.

Carvalho HF, Felisbino SL, Keene DR, and Vogel KG. 2006. Identification, content, and distribution of type VI collagen in bovine tendons. Cell Tissue Res 325:315-324.

Cattaruzza S, Ozerdem U, Denzel M, Ranscht B, Bulian P, Cavallaro U, Zanocco D, Colombatti A, Stallcup WB, and Perris R. 2013. Multivalent proteoglycan modulation of FGF mitogenic responses in perivascular cells. Angiogenesis 16:309-327. 10.1007/s10456012-9316-7

Chen XD, Allen MR, Bloomfield S, Xu T, and Young M. 2003. Biglycan-deficient mice have delayed osteogenesis after marrow ablation. Calcif Tissue Int 72:577-582. $10.1007 / \mathrm{s} 00223-002-1101-\mathrm{y}$

Choi RK, Smith MM, Martin JH, Clarke JL, Dart AJ, Little CB, and Clarke EC. 2016. Chondroitin sulphate glycosaminoglycans contribute to widespread inferior biomechanics in tendon after focal injury. J Biomech 49:2694-2701. 10.1016/j.jbiomech.2016.06.006

Chun JS, Oh H, Yang S, and Park M. 2008. Wnt signaling in cartilage development and degeneration. BMB Rep 41:485-494.

Cotman SL, Halfter W, and Cole GJ. 1999. Identification of extracellular matrix ligands for the heparan sulfate proteoglycan agrin. Exp Cell Res 249:54-64. 10.1006/excr.1999.4463

Danielson KG, Baribault H, Holmes DF, Graham H, Kadler KE, and Iozzo RV. 1997. Targeted disruption of decorin leads to abnormal collagen fibril morphology and skin fragility. $J$ Cell Biol 136:729-743.

Echtermeyer F, Bertrand J, Dreier R, Meinecke I, Neugebauer K, Fuerst M, Lee YJ, Song YW, Herzog C, Theilmeier G, and Pap T. 2009. Syndecan-4 regulates ADAMTS-5 activation and cartilage breakdown in osteoarthritis. Nat Med 15:1072-1076. 10.1038/nm.1998

Eldridge S, Nalesso G, Ismail H, Vicente-Greco K, Kabouridis P, Ramachandran M, Niemeier A, Herz J, Pitzalis C, Perretti M, and Dell'Accio F. 2016. Agrin mediates chondrocyte homeostasis and requires both LRP4 and alpha-dystroglycan to enhance cartilage formation in vitro and in vivo. Ann Rheum Dis 75:1228-1235. 10.1136/annrheumdis2015-207316

Ezura Y, Chakravarti S, Oldberg A, Chervoneva I, and Birk DE. 2000. Differential expression of lumican and fibromodulin regulate collagen fibrillogenesis in developing mouse tendons. J Cell Biol 151:779-788. 
580 Farndale RW, Buttle DJ, and Barrett AJ. 1986. Improved quantitation and discrimination of

581

582

583

584

585

586

587

588

589

590

591

592

593

594

595

596

597

598

599

600

601

602

603

604

605

606

607

608

609

610

611

612

613

614

615

616

617

618

619

620

621

622

623 sulphated glycosaminoglycans by use of dimethylmethylene blue. Biochim Biophys Acta 883:173-177. 0304-4165(86)90306-5 [pii]

Finni T. 2006. Structural and functional features of human muscle-tendon unit. Scand J Med Sci Sports 16:147-158. 10.1111/j.1600-0838.2005.00494.x

Gallagher J. 2015. Fell-Muir Lecture: Heparan sulphate and the art of cell regulation: a polymer chain conducts the protein orchestra. Int J Exp Pathol 96:203-231. 10.1111/iep.12135

Grant TM, Thompson MS, Urban J, and Yu J. 2013. Elastic fibres are broadly distributed in tendon and highly localized around tenocytes. J Anat 222:573-579. 10.1111/joa. 12048

Hausser HJ, Ruegg MA, Brenner RE, and Ksiazek I. 2007. Agrin is highly expressed by chondrocytes and is required for normal growth. Histochem Cell Biol 127:363-374. 10.1007/s00418-006-0258-2

Hayes AJ, Lord MS, Smith SM, Smith MM, Whitelock JM, Weiss AS, and Melrose J. 2011a. Colocalization in vivo and association in vitro of perlecan and elastin. Histochem Cell Biol 136:437-454. 10.1007/s00418-011-0854-7

Hayes AJ, Shu CC, Lord MS, Little CB, Whitelock JM, and Melrose J. 2016. Pericellular colocalisation and interactive properties of type VI collagen and perlecan in the intervertebral disc. Eur Cell Mater 32:40-57.

Hayes AJ, Smith SM, Gibson MA, and Melrose J. 2011b. Comparative immunolocalization of the elastin fiber-associated proteins fibrillin-1, LTBP-2, and MAGP-1 with components of the collagenous and proteoglycan matrix of the fetal human intervertebral disc. Spine (Phila Pa 1976) 36:E1365-1372. 10.1097/BRS.0b013e31821fd23e

Horikawa O, Nakajima H, Kikuchi T, Ichimura S, Yamada H, Fujikawa K, and Toyama Y. 2004. Distribution of type VI collagen in chondrocyte microenvironment: study of chondrons isolated from human normal and degenerative articular cartilage and cultured chondrocytes. J Orthop Sci 9:29-36. 10.1007/s00776-003-0737-4 [doi]

Iozzo RV. 1998. Matrix proteoglycans: from molecular design to cellular function. Annu Rev Biochem 67:609-652.

Iozzo RV, and Schaefer L. 2015. Proteoglycan form and function: A comprehensive nomenclature of proteoglycans. Matrix Biol. 10.1016/j.matbio.2015.02.003

Islam M, Gor J, Perkins SJ, Ishikawa Y, Bachinger HP, and Hohenester E. 2013. The concave face of decorin mediates reversible dimerization and collagen binding. J Biol Chem 288:35526-35533. 10.1074/jbc.M113.504530

Jensen SA, Robertson IB, and Handford PA. 2012. Dissecting the fibrillin microfibril: structural insights into organization and function. Structure 20:215-225. 10.1016/j.str.2011.12.008

Jepsen KJ, Wu F, Peragallo JH, Paul J, Roberts L, Ezura Y, Oldberg A, Birk DE, and Chakravarti S. 2002. A syndrome of joint laxity and impaired tendon integrity in lumican- and fibromodulin-deficient mice. J Biol Chem 277:35532-35540. 10.1074/jbc.M205398200 [doi] M205398200 [pii]

Kalamajski S, Aspberg A, and Oldberg A. 2007. The decorin sequence SYIRIADTNIT binds collagen type I. J Biol Chem 282:16062-16067. 10.1074/jbc.M700073200

Kim MJ, Cotman SL, Halfter W, and Cole GJ. 2003. The heparan sulfate proteoglycan agrin modulates neurite outgrowth mediated by FGF-2. J Neurobiol 55:261-277. 10.1002/neu.10213 
624 Kishimoto Y, Ohkawara B, Sakai T, Ito M, Masuda A, Ishiguro N, Shukunami C, Docheva D,

625

626

627

628

629

630

631

632

633

634

635

636

637

638

639

640

641

642

643

644

645

646

647

648

649

650

651

652

653

654

655

656

657

658

659

660

661

662

663

664

665

666

667

668

669 and Ohno K. 2017. Wnt/beta-catenin signaling suppresses expressions of Scx, Mkx, and Tnmd in tendon-derived cells. PLoS One 12:e0182051. 10.1371/journal.pone.0182051

LeBaron RG, Hook A, Esko JD, Gay S, and Hook M. 1989. Binding of heparan sulfate to type V collagen. A mechanism of cell-substrate adhesion. J Biol Chem 264:7950-7956.

Li Q, Doyran B, Gamer LW, Lu XL, Qin L, Ortiz C, Grodzinsky AJ, Rosen V, and Han L. 2015. Biomechanical properties of murine meniscus surface via AFM-based nanoindentation. $J$ Biomech 48:1364-1370. 10.1016/j.jbiomech.2015.02.064

Linsenmayer TF, Gibney E, Igoe F, Gordon MK, Fitch JM, Fessler LI, and Birk DE. 1993. Type V collagen: molecular structure and fibrillar organization of the chicken alpha $1(\mathrm{~V}) \mathrm{NH} 2-$ terminal domain, a putative regulator of corneal fibrillogenesis. J Cell Biol 121:11811189.

Liu CF, Breidenbach A, Aschbacher-Smith L, Butler D, and Wylie C. 2013. A role for hedgehog signaling in the differentiation of the insertion site of the patellar tendon in the mouse. PLoS One 8:e65411. 10.1371/journal.pone.0065411

Liu H, Zhu S, Zhang C, Lu P, Hu J, Yin Z, Ma Y, Chen X, and OuYang H. 2014. Crucial transcription factors in tendon development and differentiation: their potential for tendon regeneration. Cell Tissue Res 356:287-298. 10.1007/s00441-014-1834-8

Liu SH, Yang RS, al-Shaikh R, and Lane JM. 1995. Collagen in tendon, ligament, and bone healing. A current review. Clin Orthop Relat Res:265-278.

Liu W, Watson SS, Lan Y, Keene DR, Ovitt CE, Liu H, Schweitzer R, and Jiang R. 2010. The atypical homeodomain transcription factor Mohawk controls tendon morphogenesis. Mol Cell Biol 30:4797-4807. 10.1128/mcb.00207-10

McLeod MA, Wilusz RE, and Guilak F. 2013. Depth-dependent anisotropy of the micromechanical properties of the extracellular and pericellular matrices of articular cartilage evaluated via atomic force microscopy. J Biomech 46:586-592. 10.1016/j.jbiomech.2012.09.003

McNulty AL, and Guilak F. 2015. Mechanobiology of the meniscus. J Biomech. 10.1016/j.jbiomech.2015.02.008

Melrose J, Isaacs MD, Smith SM, Hughes CE, Little CB, Caterson B, and Hayes AJ. 2012. Chondroitin sulphate and heparan sulphate sulphation motifs and their proteoglycans are involved in articular cartilage formation during human foetal knee joint development. Histochem Cell Biol 138:461-475. 10.1007/s00418-012-0968-6

Melrose J, Smith MM, Smith SM, Ravi V, Young AA, Dart AJ, Sonnabend DH, and Little CB. 2013. Altered stress induced by partial transection of the infraspinatus tendon leads to perlecan (HSPG2) accumulation in an ovine model of tendinopathy. Tissue Cell 45:7782. 10.1016/j.tice.2012.10.001

Miyabara S, Yuda Y, Kasashima Y, Kuwano A, and Arai K. 2014. Regulation of Tenomodulin Expression Via Wnt/beta-catenin Signaling in Equine Bone Marrow-derived Mesenchymal Stem Cells. J Equine Sci 25:7-13. 10.1294/jes.25.7

Moon YL, Singh H, Yang H, and Chul LK. 2011. Arthroscopic rotator interval closure by purse string suture for symptomatic inferior shoulder instability. Orthopedics 34. 10.3928/01477447-20110228-02

Nurminskaya MV, and Birk DE. 1998. Differential expression of genes associated with collagen fibril growth in the chicken tendon: identification of structural and regulatory genes by subtractive hybridization. Arch Biochem Biophys 350:1-9. 10.1006/abbi.1997.0498 
670 Pang X, Wu JP, Allison GT, Xu J, Rubenson J, Zheng MH, Lloyd DG, Gardiner B, Wang A, and

671

672

673

674

675

676

677

678

679

680

681

682

683

684

685

686

687

688

689

690

691

692

693

694

695

696

697

698

699

700

701

702

703

704

705

706

707

708

709

710

711

712

713

714

715
Kirk TB. 2017. Three dimensional microstructural network of elastin, collagen, and cells in Achilles tendons. J Orthop Res 35:1203-1214. 10.1002/jor.23240

Petit B, Ronziere MC, Hartmann DJ, and Herbage D. 1993. Ultrastructural organization of type XI collagen in fetal bovine epiphyseal cartilage. Histochemistry 100:231-239.

Plodinec M, Loparic M, and Aebi U. 2010. Imaging articular cartilage tissue using atomic force microscopy (AFM). Cold Spring Harb Protoc 2010:pdb prot5499.

Ricard-Blum S, Beraud M, Raynal N, Farndale RW, and Ruggiero F. 2006. Structural requirements for heparin/heparan sulfate binding to type V collagen. J Biol Chem 281:25195-25204. 10.1074/jbc.M603096200

Salmivirta M, and Jalkanen M. 1995. Syndecan family of cell surface proteoglycans: developmentally regulated receptors for extracellular effector molecules. Experientia 51:863-872.

Sanchez-Adams J, Wilusz RE, and Guilak F. 2013. Atomic force microscopy reveals regional variations in the micromechanical properties of the pericellular and extracellular matrices of the meniscus. J Orthop Res 31:1218-1225. 10.1002/jor.22362

Sardone F, Santi S, Tagliavini F, Traina F, Merlini L, Squarzoni S, Cescon M, Wagener R, Maraldi NM, Bonaldo P, Faldini C, and Sabatelli P. 2016. Collagen VI-NG2 axis in human tendon fibroblasts under conditions mimicking injury response. Matrix Biol 55:90-105. 10.1016/j.matbio.2016.02.012

Sato N, Taniguchi T, Goda Y, Kosaka H, Higashino K, Sakai T, Katoh S, Yasui N, Sairyo K, and Taniguchi H. 2016. Proteomic Analysis of Human Tendon and Ligament: Solubilization and Analysis of Insoluble Extracellular Matrix in Connective Tissues. $J$ Proteome Res 15:4709-4721. 10.1021/acs.jproteome.6b00806

Sato N, Taniguchi, T, Goda, Y, Kosaka, H, Higashino, K, Sakai, T, Sairyo K, Katoh, S, Taniguchi, H, Yasui, N. 2011. Establishment of a methods for proteomic analysis of human Achilles tendon. Trans Orthop Res Soc

Sawaguchi N, Majima T, Iwasaki N, Funakoshi T, Shimode K, Onodera T, and Minami A. 2006. Extracellular matrix modulates expression of cell-surface proteoglycan genes in fibroblasts. Connect Tissue Res 47:141-148. 10.1080/03008200600685459

Schonherr E, Witsch-Prehm P, Harrach B, Robenek H, Rauterberg J, and Kresse H. 1995. Interaction of biglycan with type I collagen. J Biol Chem 270:2776-2783.

Screen HR, Berk DE, Kadler KE, Ramirez F, and Young MF. 2015. Tendon functional extracellular matrix. J Orthop Res 33:793-799. 10.1002/jor.22818

Shu C, Smith SM, and Melrose J. 2016. The heparan sulphate deficient Hspg2 exon 3 null mouse displays reduced deposition of TGF-beta1 in skin compared to C57BL/6 wild type mice. $J$ Mol Histol 47:365-374. 10.1007/s10735-016-9677-0

Smith GN, Jr., Hasty KA, and Brandt KD. 1989. Type XI collagen is associated with the chondrocyte surface in suspension culture. Matrix 9:186-192.

Smith SM, Thomas CE, and Birk DE. 2012. Pericellular proteins of the developing mouse tendon: a proteomic analysis. Connect Tissue Res 53:2-13. 10.3109/03008207.2011.602766

Smith SM, Zhang G, and Birk DE. 2014. Collagen V localizes to pericellular sites during tendon collagen fibrillogenesis. Matrix Biol 33:47-53. 10.1016/j.matbio.2013.08.003

Stegemann H, and Stalder K. 1967. Determination of hydroxyproline. Clin Chim Acta 18:267273. 
716 Svensson L, Heinegard D, and Oldberg A. 1995. Decorin-binding sites for collagen type I are

717

718

719

720

721

722

723

724

725

726

727

728

729

730

731

732

733

734

735

736

737

738

739

740

741

742

743

744

745

746

747

748

749

750

751

752

753

754

755

756

757

758

759

760

mainly located in leucine-rich repeats 4-5. J Biol Chem 270:20712-20716.

Svensson L, Narlid I, and Oldberg A. 2000. Fibromodulin and lumican bind to the same region on collagen type I fibrils. FEBS Lett 470:178-182.

Taffetani M, Raiteri R, Gottardi R, Gastaldi D, and Vena P. 2015. A quantitative interpretation of the response of articular cartilage to atomic force microscopy-based dynamic nanoindentation tests. J Biomech Eng 137. 10.1115/1.4030175

Takeuchi S, Nakano S, Nakamura K, Ozoe A, Chien P, Yoshihara H, Hakuno F, Matsuwaki T, Saeki Y, Takahashi S, Yamanouchi K, and Nishihara M. 2016. Roles of chondroitin sulfate proteoglycan 4 in fibrogenic/adipogenic differentiation in skeletal muscle tissues. Exp Cell Res 347:367-377. 10.1016/j.yexcr.2016.08.023

Tang F, Lord MS, Stallcup WB, and Whitelock JM. 2018. Cell surface chondroitin sulfate proteoglycan 4 (CSPG4) binds to the basement membrane heparan sulfate proteoglycan, perlecan, and is involved in cell adhesion. J Biochem. $10.1093 / \mathrm{jb} / \mathrm{mvy} 008$

van den Berg WB. 2011. Osteoarthritis year 2010 in review: pathomechanisms. Osteoarthritis Cartilage 19:338-341. 10.1016/j.joca.2011.01.022

Viola M, Bartolini B, Sonaggere M, Giudici C, Tenni R, and Tira ME. 2007. Fibromodulin interactions with type I and II collagens. Connect Tissue Res 48:141-148. $10.1080 / 03008200701276133$

Walz DM, Burge AJ, and Steinbach L. 2015. Imaging of shoulder instability. Semin Musculoskelet Radiol 19:254-268. 10.1055/s-0035-1549319

Wang JH, Guo Q, and Li B. 2012a. Tendon biomechanics and mechanobiology--a minireview of basic concepts and recent advancements. J Hand Ther 25:133-140; quiz 141. 10.1016/j.jht.2011.07.004

Wang M, Peng Z, Watson JA, Watson GS, and Yin L. 2012b. Nanoscale study of cartilage surfaces using atomic force microscopy. Proc Inst Mech Eng H 226:899-910. 10.1177/0954411912460482

Wenstrup RJ, Florer JB, Brunskill EW, Bell SM, Chervoneva I, and Birk DE. 2004. Type V collagen controls the initiation of collagen fibril assembly. J Biol Chem 279:5333153337. 10.1074/jbc.M409622200

Wenstrup RJ, Smith SM, Florer JB, Zhang G, Beason DP, Seegmiller RE, Soslowsky LJ, and Birk DE. 2011. Regulation of collagen fibril nucleation and initial fibril assembly involves coordinate interactions with collagens $\mathrm{V}$ and XI in developing tendon. $J$ Biol Chem 286:20455-20465. 10.1074/jbc.M111.223693

Whitelock J, and Melrose J. 2011. Heparan sulfate proteoglycans in healthy and diseased systems. Wiley Interdiscip Rev Syst Biol Med 3:739-751. 10.1002/wsbm.149

Whitelock JM, and Iozzo RV. 2005. Heparan sulfate: a complex polymer charged with biological activity. Chem Rev 105:2745-2764.

Whitelock JM, Melrose J, and Iozzo RV. 2008. Diverse cell signaling events modulated by perlecan. Biochemistry 47:11174-11183.

Wilusz RE, Defrate LE, and Guilak F. 2012. A biomechanical role for perlecan in the pericellular matrix of articular cartilage. Matrix Biol 31:320-327.

10.1016/j.matbio.2012.05.002

Wilusz RE, Sanchez-Adams J, and Guilak F. 2014. The structure and function of the pericellular matrix of articular cartilage. Matrix Biol 39:25-32. 10.1016/j.matbio.2014.08.009 
761 Yamada S, Sugahara K, and Ozbek S. 2011. Evolution of glycosaminoglycans: Comparative $762 \quad$ biochemical study. Commun Integr Biol 4:150-158. 10.4161/cib.4.2.14547

$763 \mathrm{Yu}$ J, and Urban JP. 2010. The elastic network of articular cartilage: an immunohistochemical $764 \quad$ study of elastin fibres and microfibrils. J Anat 216:533-541. 10.1111/j.1469765 7580.2009.01207.x

766 Zelenski NA, Leddy HA, Sanchez-Adams J, Zhang J, Bonaldo P, Liedtke W, and Guilak F. 767 2015a. Collagen VI regulates pericellular matrix properties, chondrocyte swelling, and mechanotransduction in articular cartilage. Arthritis Rheumatol. 10.1002/art.39034

Zelenski NA, Leddy HA, Sanchez-Adams J, Zhang J, Bonaldo P, Liedtke W, and Guilak F. 2015b. Type VI Collagen Regulates Pericellular Matrix Properties, Chondrocyte Swelling, and Mechanotransduction in Mouse Articular Cartilage. Arthritis Rheumatol 67:1286-1294. 10.1002/art.39034

Zhou Z, Wang J, Cao R, Morita H, Soininen R, Chan KM, Liu B, Cao Y, and Tryggvason K. 2004. Impaired angiogenesis, delayed wound healing and retarded tumor growth in perlecan heparan sulfate-deficient mice. Cancer Res 64:4699-4702. 


\section{Table 1 (on next page)}

Murine-specific primers

These primers were designed using MacVector for real time PCR 
1 Table 1. Murine-specific primers designed using MacVector for real time PCR

2

\begin{tabular}{|c|c|c|c|c|}
\hline Molecule (gene) & $\begin{array}{c}\text { Mouse } \\
\text { Accession \# }\end{array}$ & Sequence 5' to 3' & $\begin{array}{l}\text { Annealing } \\
\text { Temp }\left({ }^{\circ} \mathrm{C}\right)\end{array}$ & $\begin{array}{l}\text { Product } \\
\text { size (bp) }\end{array}$ \\
\hline \multicolumn{5}{|c|}{ Extracellular matrix proteins } \\
\hline Collagen I (Colla1) & X06753 & $\begin{array}{l}\text { F } \text { TCT CCA CTC TTC TAG TTC CT } \\
R\end{array}$ & 55 & 269 \\
\hline Versican (Vcan) & XM994074 & $\begin{array}{l}\mathrm{F} \text { ATG ATG GGG AAG GAA GGG GTT C } \\
\mathrm{R} \text { AGC CAG CCG TAA TCG CAT TG }\end{array}$ & 57 & 236 \\
\hline $\operatorname{Biglycan}(B g n)$ & L20276 & $\begin{array}{l}\mathrm{F} \text { ACT TCT GTC CTA TGG GCT TCG G } \\
\mathrm{R} \text { GCT TCT TCA TCT GGC TAT GTT CCT C }\end{array}$ & 57 & 218 \\
\hline $\operatorname{Lumican}($ Lum $)$ & NM008524 & $\begin{array}{ll}\mathrm{F} & \text { TAC AAC AAC CTG ACC GAG TCC G } \\
\mathrm{R} & \text { CGA GAC AGC ATC CTC TTT GAG C }\end{array}$ & 55 & 159 \\
\hline $\operatorname{Decorin}(D c n)$ & NM007833 & $\begin{array}{ll}\mathrm{F} & \text { CAA CAA CAA ACT CCT CAG GGT GC } \\
\mathrm{R} & \text { TTG CCG TAA AGA CTC ACA GCC G } \\
\end{array}$ & 57 & 165 \\
\hline \multicolumn{5}{|c|}{ Elastin and associated proteins } \\
\hline Perlecan (Hspg2) & NM008305 & $\begin{array}{ll}\mathrm{F} & \text { TCT GTC TGC CTG GCT TCT CT } \\
\mathrm{R} & \text { CGA ATT CAA TTG TCT CGG GT }\end{array}$ & 56 & 204 \\
\hline LTBP-1 (Ltbp 1) & NM019919 & $\begin{array}{l}\text { F GGG AGC ATC TGA GTG AGG AG } \\
\text { R TCA CAG GGA TAT TGC ACA GC }\end{array}$ & 56 & 165 \\
\hline LTBP-2 (Ltbp2) & NM13589.3 & $\begin{array}{l}\mathrm{F} \text { CAC CCA GAC CAG CCT TCC CA } \\
\mathrm{R} \text { AGT CCT TGC AGA GGC CCA GG }\end{array}$ & 57 & 126 \\
\hline Fibrillin-1 $(F b n 1)$ & NM007993 & $\begin{array}{l}\text { F ATC CGC TGT ATG AAT GGG GG } \\
\text { R CTG GCA CAT CTG GTT GCT TAC C }\end{array}$ & 58 & 228 \\
\hline Elastin $(E \ln )$ & NM007925.3 & $\begin{array}{l}\text { F AGC CAA ATA TGG TGC TGC TG } \\
\text { R GGG TCC CCA GAA GAT CAC TT }\end{array}$ & 58 & 246 \\
\hline \multicolumn{5}{|l|}{ MMPs/TIMPs } \\
\hline MMP-2 (Mmp2) & NM008610 & $\begin{array}{l}\text { F ATT TGG CGG ACA GTG ACA CCA C } \\
\text { R ATC TAC TTG CTG GAC ATC AGG GGG }\end{array}$ & 59 & 231 \\
\hline MMP-3 (Mmp3) & NM010809.1 & $\begin{array}{l}\text { F GCT GAG GAC TTT CCA GGT GTT G } \\
\text { R GGT CAC TTT TTT GGC ATT TGG GTC }\end{array}$ & 53 & 120 \\
\hline MMP-13 (Mmp13) & NM008607 & $\begin{array}{l}\text { F GAT GAC CTG TCT GAG GAA G } \\
\text { R ATC AGA CCA GAC CTT GAA G }\end{array}$ & 55 & 357 \\
\hline $\begin{array}{l}\text { ADAMTS-4 } \\
\text { (Adamts4) }\end{array}$ & NM172845 & $\begin{array}{l}\text { F TAA CTT GAA TGG GCA GGG GGG TTC } \\
\text { R AAT GGC TTG AGT CAG GAC CGA AGG }\end{array}$ & 60 & 245 \\
\hline TIMP-1 (Timp1) & $\mathrm{BC} 051260$ & $\begin{array}{l}\text { F ATC TCT GGC ATC TGG CAT CCT C } \\
\text { R GGT GGT CTC GTT GAT TTC TGG G }\end{array}$ & 56 & 154 \\
\hline TIMP-3 (Timp3) & NM011595 & $\begin{array}{l}\text { F ATT ACC GCT ACC ACC TGG GTT G } \\
\text { R TCT GGG AAG AGT TAG TGT CTG GGA C }\end{array}$ & 58 & 198 \\
\hline GAPDH (Gapdh) & BC083149 & $\begin{array}{l}\mathrm{F} \text { TGC GAC TTC AAC AGC AAC TC } \\
\mathrm{R} \text { CCT GCT CAG TGT CCT TGC TG }\end{array}$ & 55 & 200 \\
\hline
\end{tabular}


Figure 1

Genomic organisation, weights of perlecan exon 3 null and wild type mice and HS contents of perlecan from the two mouse genotypes

Genomic organisation of exons 2-5 of the WT and Hspg2 $2^{\Delta-/ \Delta 3-}$ mice (A). Body weights of male WT (open symbols) and Hspg $2^{\Delta 3-/ \Delta 3-}$ mice (closed symbols) from 10-20 weeks of age (B). ELISA analysis of perlecan GAG side chains using MAb 10E4 to native HS and MAb $3 G 10$ to the $\triangle \mathrm{HS}$ stub epitope generated by heparitinase III pre-digestion demonstrating an absence of HS in the mutant perlecan. (C).

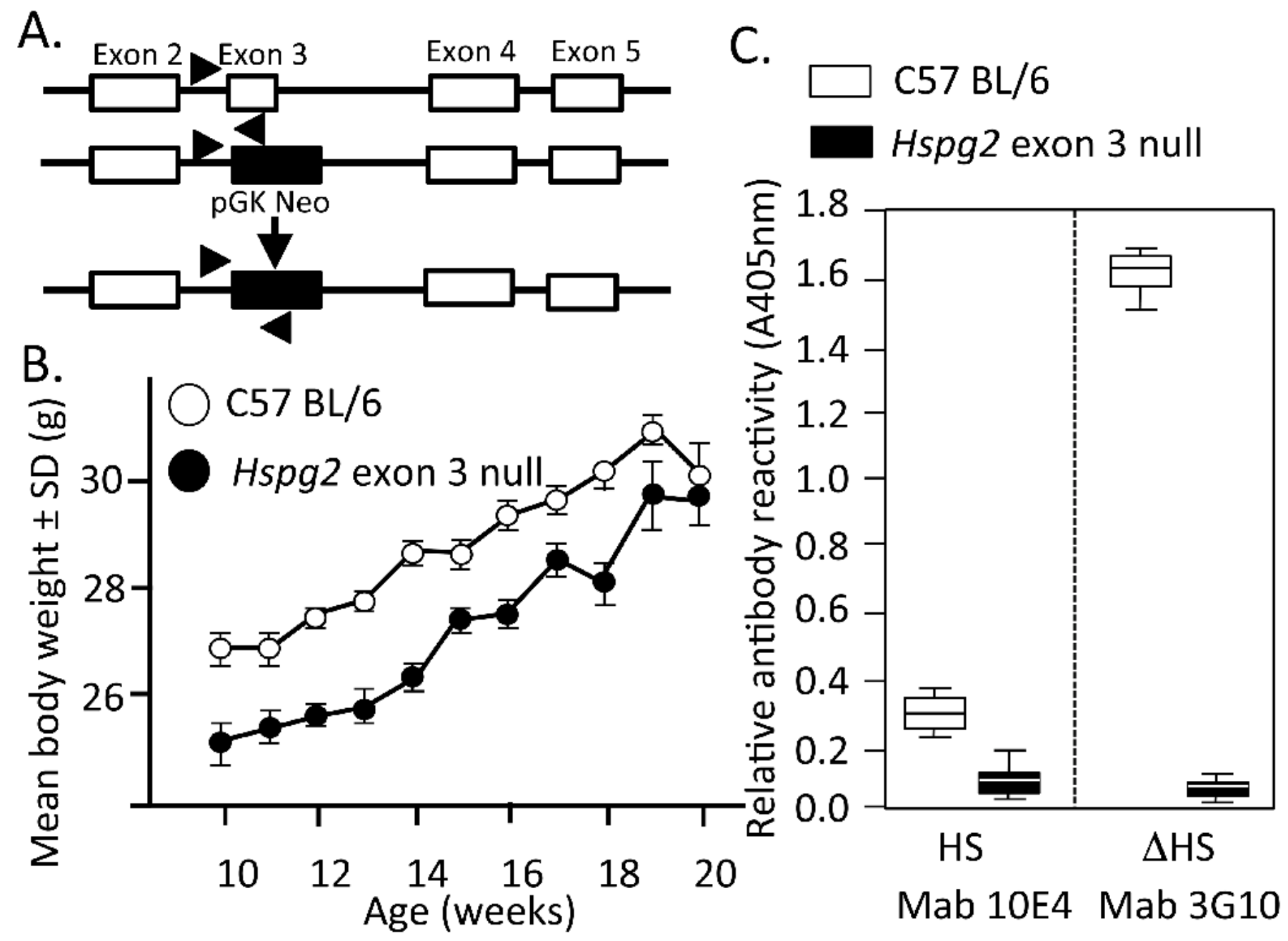




\section{Figure 2}

Material properties of wild type and perlecan exon 3 null mouse tail and Achiles tendons and compositions.

Tendon material properties: ultimate tensile stress (A, C) and tensile modulus (B, D). GAG (E) and Hydroxyproline (F) content of 3 to 12 week-old WT and Hspg2 $2^{23 / 2 / 23}$ tail tendons. Box plots show mean (line in box) and data range (box $25-75 \%$, whiskers maximum-minimum). White bars: WT; Gray bars: Hspg2 ${ }^{23-1 / 23}$. Bar graph shows mean \pm standard error of mean. Brackets $P<0.05$ between samples, $* P<0.05$ between genotypes. $N=6-8$ for each sample. 


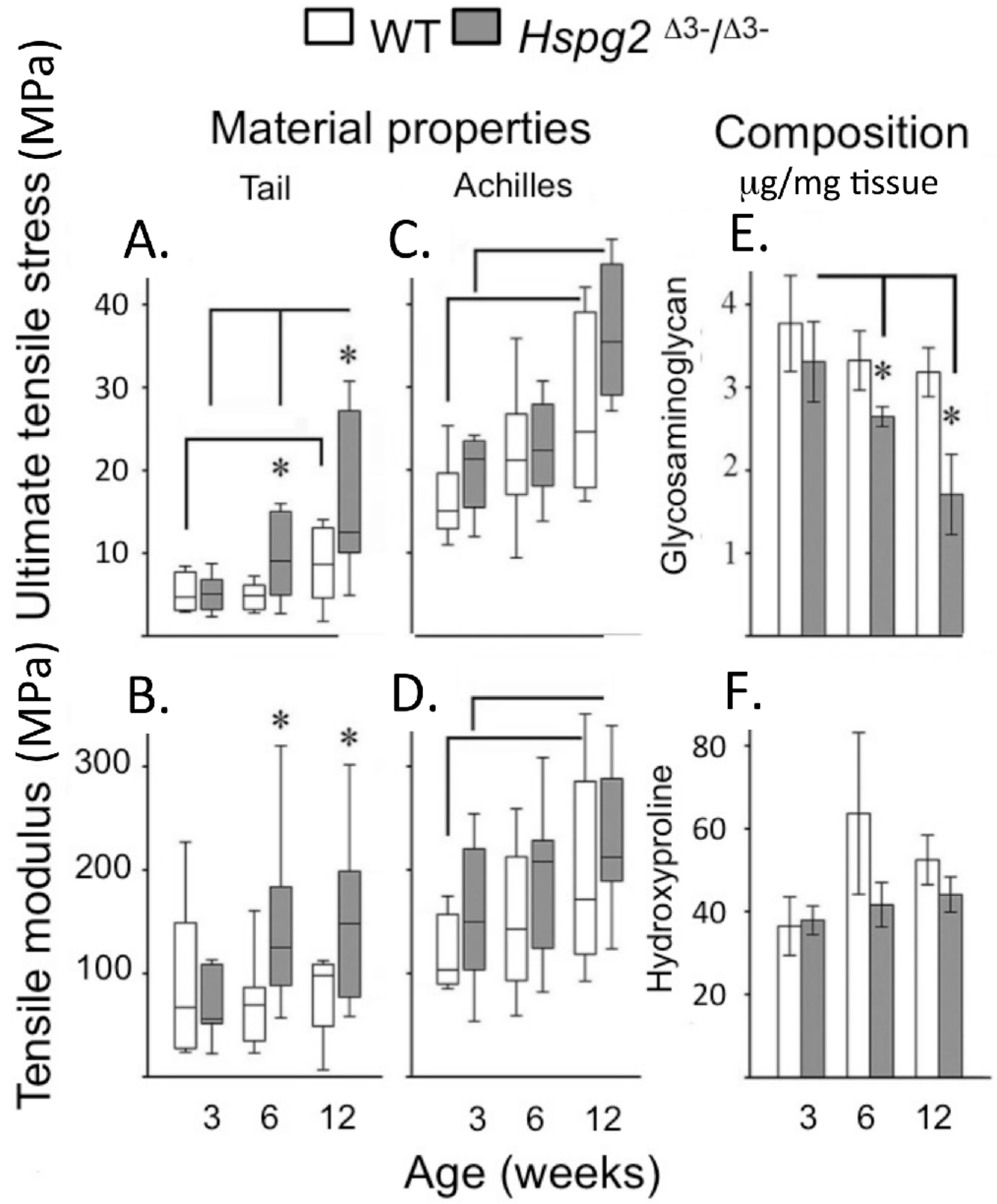




\section{Figure 3}

Material properties of Achilles tendons following tenotomy and recovery for up to 8 weeks post surgery.

Achilles tendon material properties at 2, 4 and 8 weeks after surgical tenotemy: normal contralateral $(A, C)$ and surgical tenotomy (B, D), equivalent to 14,16 and 20 weeks of age. White bars: WT; Gray bars: Hspg2 ${ }^{23-133}$. Box plot show median (line in box), inter-quartile range (box) and data range (whiskers, maximum - minimum). Bracket $-P<0.05$ between samples. $* P<0.05$ between genotypes. $\# P<0.05$ to contralateral. $N=6-8$. 


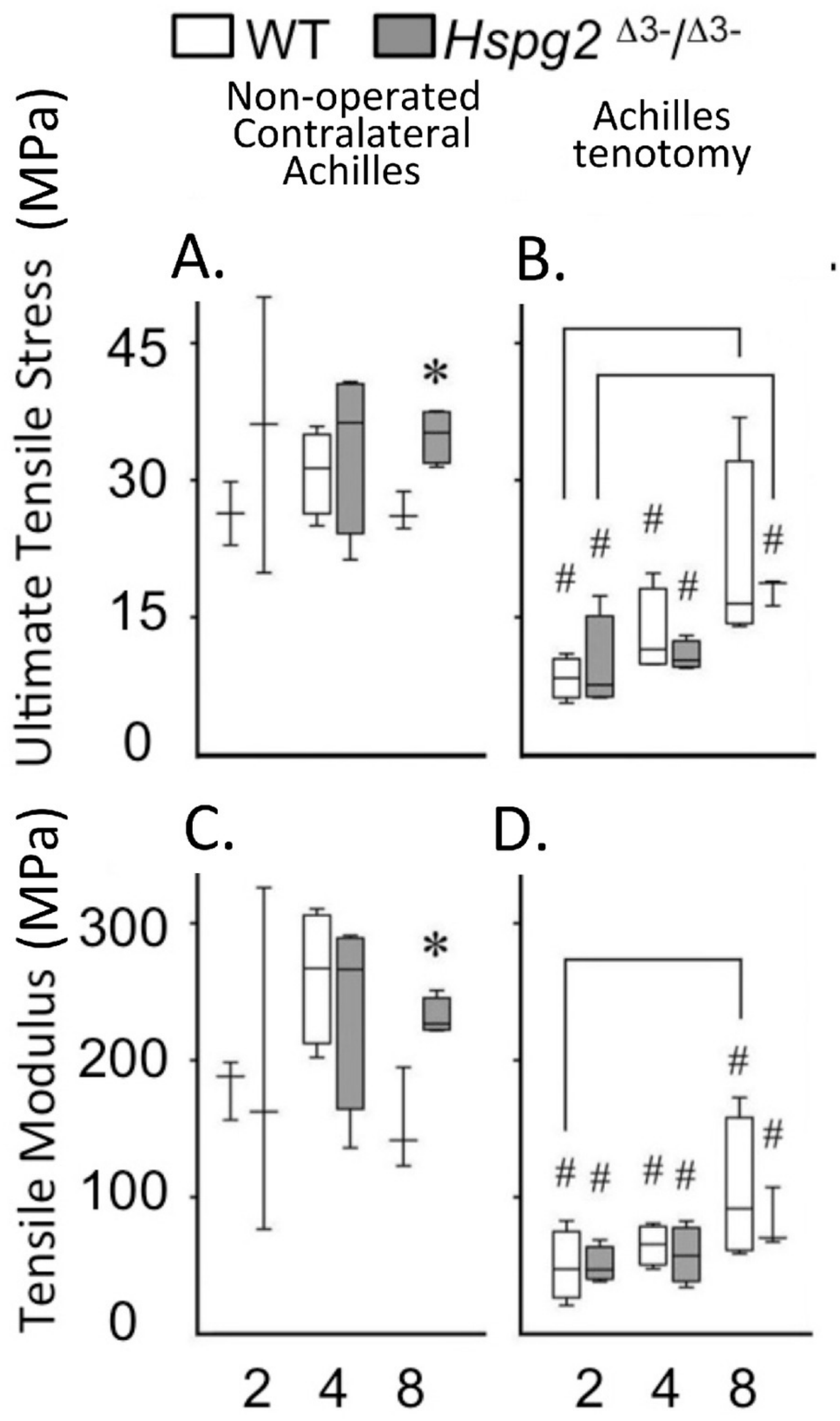

Peer) reviewing PDF I (2) 
Figure 4

Comparative gene expression in wild type and perlecan exon 3 mouse tail tendon.

Comparative gene expression of selected extracellular matrix genes and elastin-associated protein genes in mouse ex vivo tail tendons at 3, 6 and 12 weeks old. $* P<0.05$ between genotype. Data were normalised to Gapdh expression. White bars: WT; Gray bars: Hspg2 ${ }^{23-1 / 33 .}$. Box plot shows mean (line in box), interquartile range (box) and data range (whiskers, maximum - minimum). $\mathrm{N}=6$ per sample.

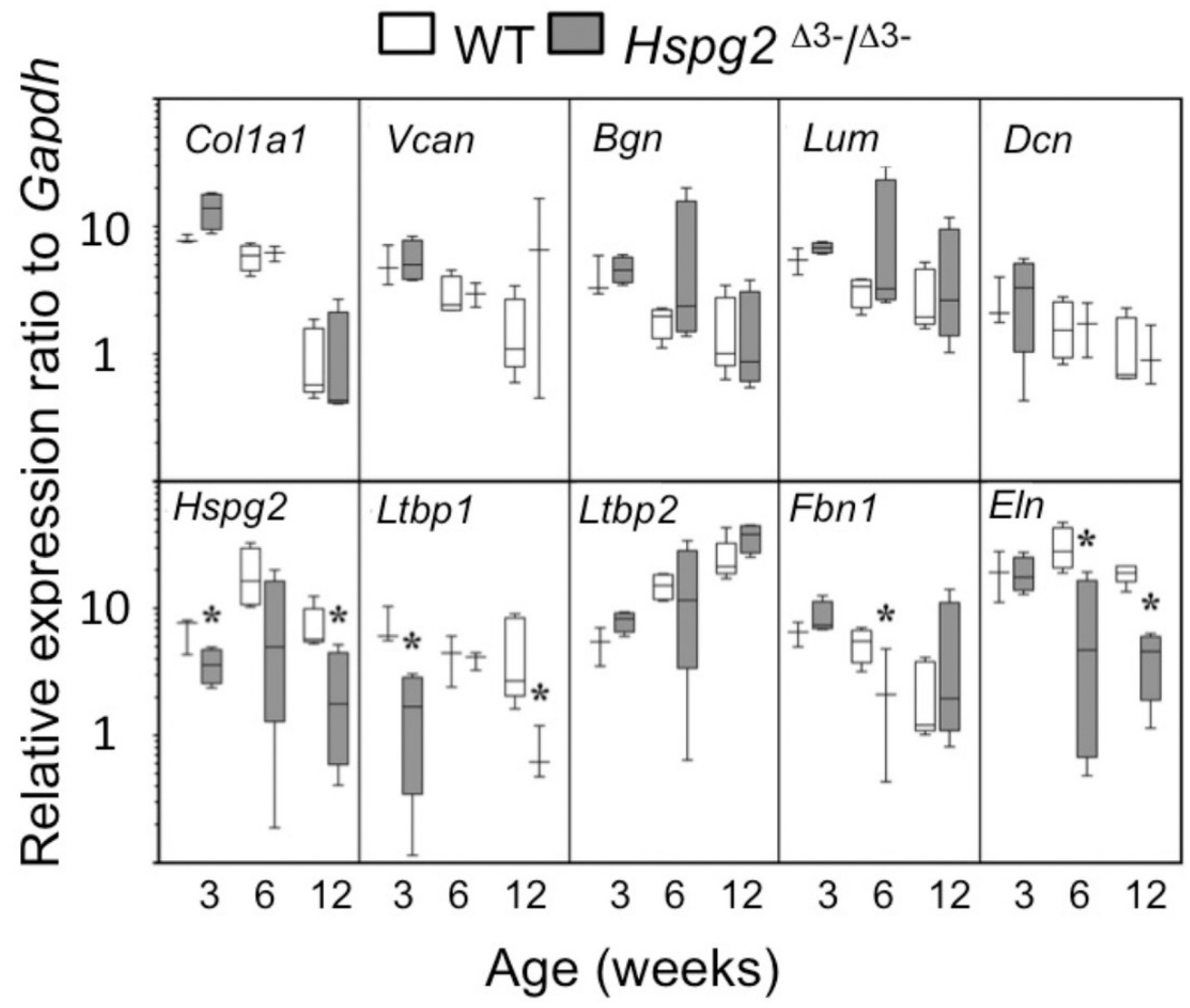




\section{Figure 5}

Influence of FGF-2 on cultured wild type and perlecan exon 3 null tail tenocytes in monolayer culture

Gene expression in 3 week-old mouse tail tendon outgrowth tenocytes cultured with FGF-2 $(0,1,10$ or $100 \mathrm{ng} / \mathrm{ml})$. Data expressed as a fold change relative to the expression of the WT control (0 ng/ml FGF-2). White bars: WT; Gray bars: Hspg2 $2^{\Delta 3-133}$. Box plot shows mean (line in box), interquartile range (box) and maximum - minimum (whiskers). $\mathrm{N}=6$ for each sample. $\# P<0.05$ compared to untreated control; $* P<0.05$ between genotypes at that concentration of FGF-2. Note Mmp13 Y-axis differs from all other genes with range from 0.1$10000 x$ instead of $0.1-100 x$. 


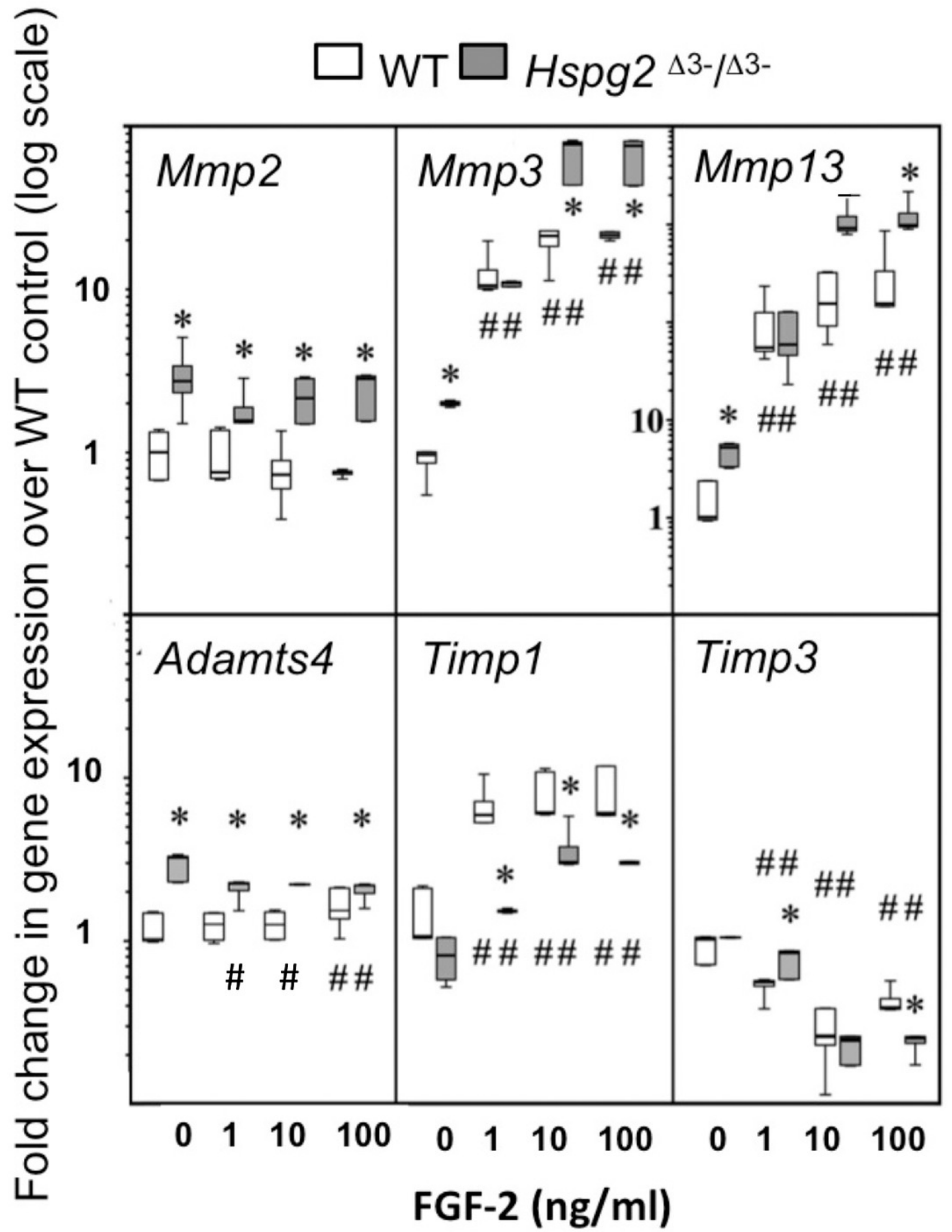




\section{Figure 6}

Measurement of the collagen fibril diameters in wild type and perlecan exon 3 null tail and Achilles tendons by transmission electron microscopy.

Measurement of the collagen fibril diameters in cross-section of 3 and 12 week-old mouse tail and Achilles tendons from transmission electron microscopy (TEM) images. Representative TEM images $(A, B)$ are shown with respective mean \pm standard deviations. Fibril diameter data were categorised into defined nanometer ranges ( $x$ axis) giving the overall size distribution (C, D). The frequencies were expressed as a percentage of the total fibril numbers counted (y axis). Three tendon samples from three animals per age/genotype were measured. Bar graph shows mean \pm standard deviation. White bars: WT; Gray bars: Hspg $2^{23-}$ $1 \Delta 3$ 


\section{$\square$ WT $\square$ Hspg2 ${ }^{\Delta 3-/ \Delta 3-}$}

\section{Tail Tendon}

3 wks

Wild Type Hspg2 ${ }^{\Delta 3-/ \Delta 3-}$

12 wks

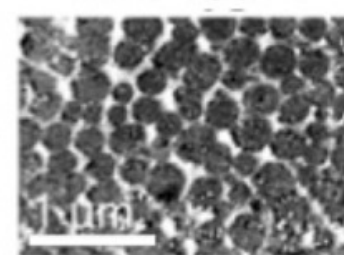

Mean $202 \pm 60 \mathrm{~nm} 193+46 \mathrm{~nm}$
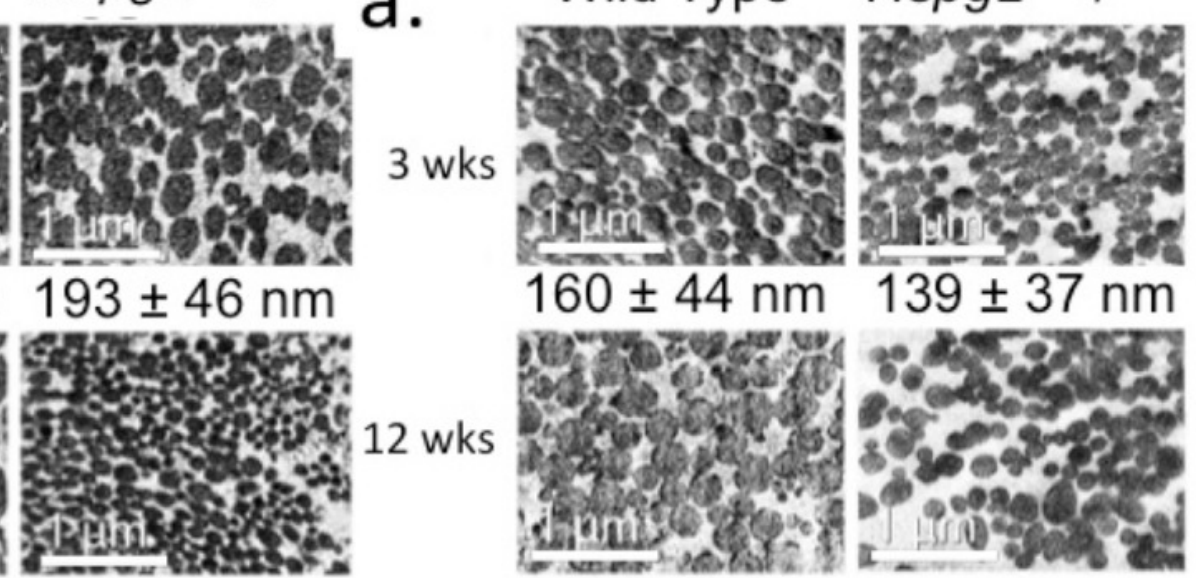

Mean $243 \pm 81 \mathrm{~nm} \quad 84 \pm 28 \mathrm{~nm}$

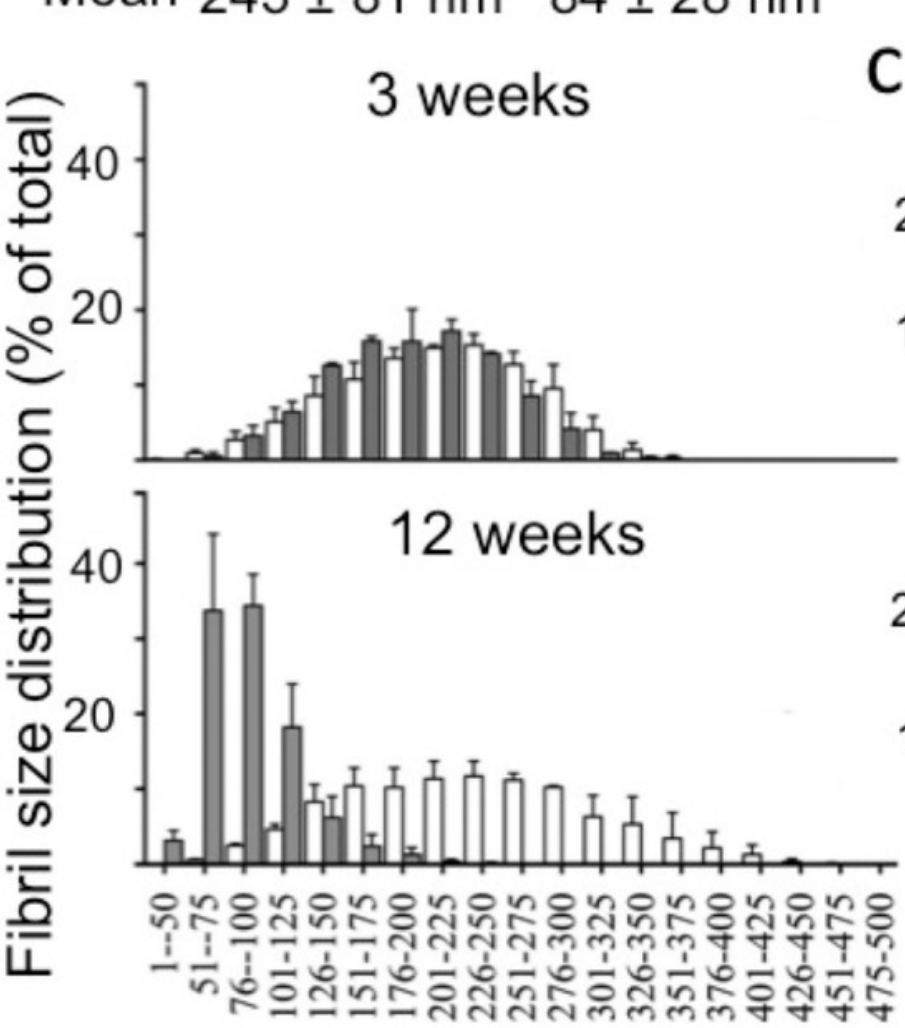

a.

3 wks

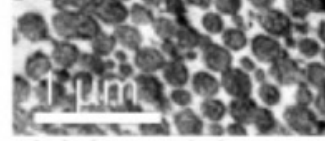

$160 \pm 44 \mathrm{~nm} \quad 139 \pm 37 \mathrm{~nm}$

AchillesTendon

Wild Type Hspg2 $2^{\Delta 3-\mu \Delta-}$ b.

12 wks
C.

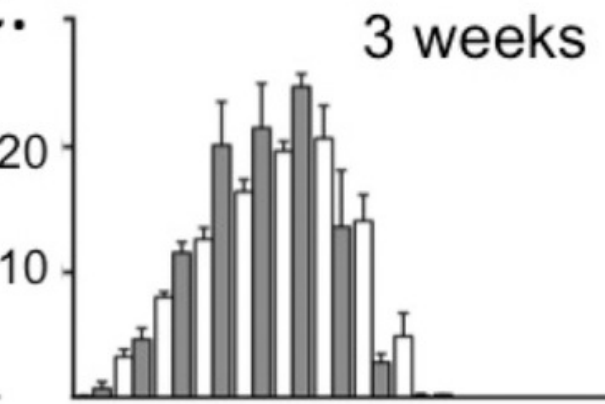

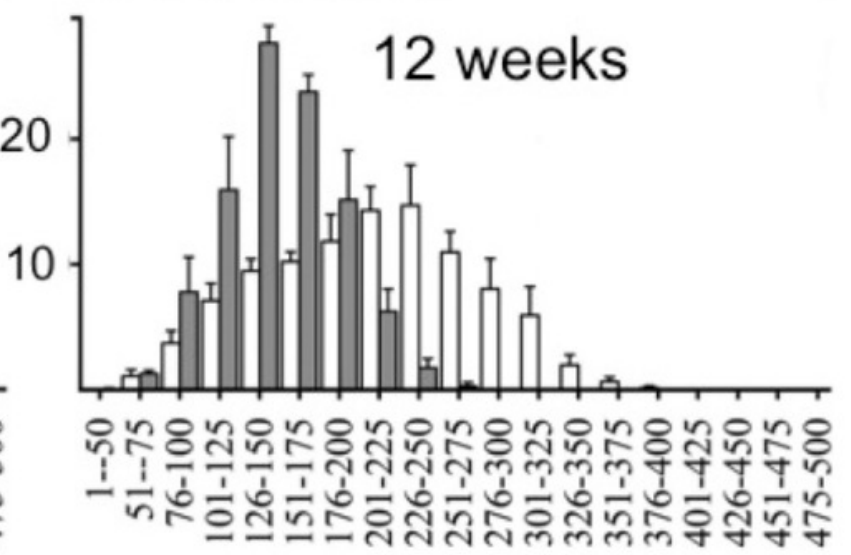

d.

Collagen fibril diameter $(\mathrm{nm})$ 